\title{
STOCHASTIC SEQUENCES WITH A REGENERATIVE STRUCTURE THAT MAY DEPEND BOTH ON THE FUTURE AND ON THE PAST
}

\author{
SERGEY FOSS, ${ }^{* * *}$ Heriot-Watt University and Sobolev Institute of Mathematics \\ STAN ZACHARY, ${ }^{* * *}$ Heriot-Watt University
}

\begin{abstract}
Many regenerative arguments in stochastic processes use random times which are akin to stopping times, but which are determined by the future as well as the past behaviour of the process of interest. Such arguments based on 'conditioning on the future' are usually developed in an ad-hoc way in the context of the application under consideration, thereby obscuring the underlying structure. In this paper we give a simple, unified, and more general treatment of such conditioning theory. We further give a number of novel applications to various particle system models, in particular to various flavours of contact processes and to infinite-bin models. We give a number of new results for existing and new models. We further make connections with the theory of Harris ergodicity.
\end{abstract}

Keywords: Regenerative process; break point; dependence on the future and on the past; contact process; infinite-bin model; Harris ergodicity

2010 Mathematics Subject Classification: Primary 60K05; 60K35; 60K40; 60J05;

60G40; 60F99

\section{Introduction}

Many arguments in stochastic processes use random times akin to stopping times to establish regenerative or ergodic behaviour. These may be randomised stopping times as in the theory of Harris ergodicity. Alternatively, they may be random times in which there is an element of probabilistic conditioning on the, possibly infinite, future of the process of interest, but in which this conditioning is sufficiently controlled that, with respect to these random times, the process behaves as if they were stopping times; such times are used, for example, in establishing the long-term behaviour of particle systems and population processes conditional on their survival, and in establishing the behaviour of processes conditioned to avoid given regions of their state spaces. More generally, such random times, defined by conditioning on future behaviour, may also be used to establish the unconditional behaviour of their parent processes-as we illustrate in the applications of Sections 3 and 4. Such arguments based on 'conditioning on the future' are usually developed from scratch and in an ad-hoc way in the context of the application under consideration, thereby to some extent obscuring the underlying structure.

Our aim in the present paper is to give a unified treatment of these phenomena. In doing so we develop a simple theory which is more general than the sum of those already existing, and

Received 13 December 2012; revision received 18 March 2013.

* Postal address: School of Mathematics and Computer Sciences and the Maxwell Institute for Mathematical Sciences, Heriot-Watt University, Edinburgh EH14 4AS, UK.

Research of both authors was partially supported by EPSRC grant EP/I017054/1.

** Email address: s.foss@hw.ac.uk

*** Email address: s.zachary@gmail.com 
which has applications - for example, to some variants of the models considered in Sections 3 and 4-which cannot be managed by the simple application of such bits of theory as already exist. We further apply the results obtained to a number of new models, including variants of the three-state contact process of Section 3 and of the infinite-bin models of Section 4.

For simplicity of exposition, we work in this paper in discrete time. In general, the process of interest $\left\{X_{n}\right\}_{n \geq 0}$, say, may be constructed as a functional of an underlying process $\left\{\xi_{n}\right\}_{n \geq 1}$, or $\left\{\xi_{n}\right\}_{n \in \mathbb{Z}}$, where, as usual, $\mathbb{Z}$ is the set of integers. In the present paper we assume that the process $\left\{\xi_{n}\right\}$ consists of independent, identically distributed (i.i.d.) random variables $\xi_{n}$. However, some of the phenomena studied here for the process $\left\{X_{n}\right\}$ continue to occur under more relaxed assumptions for the underlying process $\left\{\xi_{n}\right\}$, for example, that it is regenerative in the sense that there are some random times at which the process starts anew independently of the past. These extensions are typically straightforward; for example, in the case where the process $\left\{\xi_{n}\right\}$ is regenerative we may restrict arguments to the regeneration times. Extensions to continuous time are also straightforward provided that the process $\left\{\xi_{n}\right\}$ is replaced by something satisfying analogous homogeneity and independence conditions; in the case of interacting particle systems (see Section 3) this is typically the collection of underlying Poisson processes.

In Section 2 we present our basic theory. Our aim is to identify sequences of random times $0 \leq \tau_{0}<\tau_{1}<\cdots$, the definition of each of which may depend both on the (typically infinite) past and on the (typically infinite) future, but which are nevertheless such that the segments of the process $\left\{\xi_{n}\right\}$ between successive such times are i.i.d. Following Kuczek [19], we shall refer to these times (which are an instance of regeneration times) as break times.

It is helpful to give an immediate example (in which the dependence is on the future only).

Example 1. Let $\left\{\xi_{n}\right\}_{n \geq 1}$ consist of i.i.d. random variables, with common distribution given by

$$
\mathbb{P}\left(\xi_{i}=1\right)=p, \quad \mathbb{P}\left(\xi_{i}=-1\right)=q, \quad \mathbb{P}\left(\xi_{i}=0\right)=1-p-q,
$$

where $0<q<p$ and $p+q<1$. We consider three variant constructions of random times whose definitions involve conditioning on the (infinite) future.

(a) For each $n \geq 0$, let $F_{n}$ be the 'future' event that $\sum_{i=1}^{m} \xi_{n+i} \geq 0$ for all $m \geq 1$. Note that the common probability of the events $F_{n}$ is strictly positive. Let $0 \leq \tau_{0}<$ $\tau_{1}<\cdots$ be the successive times $n$ at which the event $F_{n}$ occurs. Then it is easy to see (and is a special case of the result of Example 2 below) that the successive segments $\left\{\xi_{\tau_{k}+1}, \ldots, \xi_{\tau_{k+1}}\right\}, k \geq 0$, of the process $\left\{\xi_{n}\right\}$ are i.i.d. in $k$. In particular, the successive time intervals $\tau_{k+1}-\tau_{k}, k \geq 0$, are i.i.d.

(b) Now suppose that, for each $n \geq 0$, we let $F_{n}^{\prime}$ be the future event that $\sum_{i=1}^{m} \xi_{n+i} \geq 0$ for all $m \geq 1$ and, additionally, $\xi_{n+2}=0$. Again, the common probability of the events $F_{n}^{\prime}$ is strictly positive. Let $0 \leq \tau_{0}^{\prime}<\tau_{1}^{\prime}<\cdots$ be the successive times $n$ at which the event $F_{n}^{\prime}$ occurs. In this case we do not have independence of the successive segments $\left\{\xi_{\tau_{k}^{\prime}+1}, \ldots, \xi_{\tau_{k+1}^{\prime}}\right\}, k \geq 0$; for example, if the events $F_{0}^{\prime}$ and $F_{1}^{\prime}$ both occur, then necessarily $\xi_{\tau_{1}^{\prime}+1}=\xi_{2}=0$.

(c) Finally, suppose that the events $F_{n}^{\prime}$ are as in (b). However, define the sequence $0 \leq$ $\tau_{0}^{\prime \prime}<\tau_{1}^{\prime \prime}<\cdots$ by $\tau_{0}^{\prime \prime}=\min \left\{n \geq 0: F_{n}^{\prime}\right.$ occurs $\}$ (i.e. $\tau_{0}^{\prime \prime}=\tau_{0}^{\prime}$ ) and, for $k \geq 1, \tau_{k}^{\prime \prime}=$ $\min \left\{n \geq \tau_{k-1}^{\prime \prime}+2: F_{n}^{\prime}\right.$ occurs $\}$. Then it is again easy to see that the successive segments $\left\{\xi_{\tau_{k}^{\prime \prime}+1}, \ldots, \xi_{\tau_{k+1}^{\prime \prime}}\right\}, k \geq 0$, of the process $\left\{\xi_{n}\right\}$ are once more i.i.d. in $k$.

The reason for the different behaviours in the above example is that, in order to obtain i.i.d. behaviour, we require the definitions of the successive times $\tau_{k}$ to satisfy a form of monotonicity 
condition in which, in a sense which we make clear in Section 2, information about the future does not cumulate; this condition is satisfied in variants (a) and (c) of Example 1, but not in variant (b). In Section 2 we develop the relevant theory in a general setting in which the break times $\tau_{k}$ may depend on both the past and future behaviours of the underlying process $\left\{\xi_{n}\right\}$. In particular, we give conditions for the segments of the process $\left\{\xi_{n}\right\}$ between break times to constitute i.i.d. cycles. We believe this theory to be novel in the general setting. As a simple example, we apply the theory to a general random walk with positive drift (generalising Example 1).

In Section 3 we give applications of our theory to a number of discrete-time contact process models, both showing how existing results are more readily understood, and giving some new results for a three-state contact process. The theory is equally applicable in the continuoustime setting, and has applications in general to particle systems and similar models in which processes 'survive' with probabilities strictly between 0 and 1 . In Section 4 we give applications of the theory of Section 2 to a class of 'infinite-bin' models.

In Section 5 we make some connections with the existing theory of Harris-ergodic Markov chains. Finally, in Section 6 we discuss a number of other models and extended applications, including conditioning, scaling, and regeneration/asymptotic stationarity of the driving sequence $\left\{\xi_{n}\right\}$.

\section{Conditioning on the future}

We assume that the underlying process $\left\{\xi_{n}\right\}_{n \in \mathbb{Z}}$ (defined on some underlying probability space $(\Omega, \mathcal{F}, \mathbb{P}))$ consists of i.i.d. random variables $\xi_{n}$. For two events $A$ and $B$, we write $A=B$ if their symmetric difference, $A \triangle B=A \backslash B \cup B \backslash A$, has probability 0.

For $m \leq n$, denote by $\sigma_{m, n}$ the $\sigma$-algebra generated by $\xi_{m}, \ldots, \xi_{n}$, and let $\sigma_{n}=\sigma_{-\infty, n}$. The process $\left\{X_{n}\right\}_{n \in Z}$ (or $\left\{X_{n}\right\}_{n \in \mathbb{Z}_{+}}$) of interest will typically be defined in terms of the process $\left\{\xi_{n}\right\}_{n \in \mathbb{Z}}$ and adapted with respect to the filtration $\left\{\sigma_{n}\right\}_{n \in Z}$; for example, it may be defined by the stochastic recursion

$$
X_{n+1}=f\left(X_{n}, \xi_{n+1}\right)
$$

for some function $f$ (and, hence, homogeneous Markov).

Define also $\sigma$ to be the $\sigma$-algebra generated by all the random variables $\xi_{n},-\infty<n<\infty$. As usual, we may introduce a measure-preserving shift transformation $\theta$ on $\sigma$-measurable random variables by assuming that $\xi_{n} \circ \theta=\xi_{n+1}$ for all $n$, and that, more generally, $g\left(\xi_{m}, \ldots, \xi_{n}\right) \circ$ $\theta=g\left(\xi_{m+1}, \ldots, \xi_{n+1}\right)$ for any measurable function $g$. (Here the finite sequence of random variables $\left\{\xi_{m}, \ldots, \xi_{n}\right\}$ may also be replaced a half-infinite or an infinite sequence.)

We may further extend the shift transformation to events in $\sigma$ by defining (with a slight abuse of notation) $G_{1} \circ \theta=G_{2}$ if $\mathbf{1}_{G_{1}} \circ \theta=\mathbf{1}_{G_{2}}$. (Here by $\mathbf{1}_{G_{1}}$ we denote the indicator function of the event $G_{1}$ which equals 1 if the event $G_{1}$ occurs and 0 otherwise). We then say that a sequence of events $\left\{G_{n}\right\}$ is stationary if it is so with respect to $\theta$, i.e. $G_{n} \circ \theta=G_{n+1}$ for all $n$. Therefore, if there are two sequences and each of them is stationary, then they are also jointly stationary.

In what follows, we consider, in addition to the process $\left\{\xi_{n}\right\}$, a given sequence of events $\left\{F_{n}\right\}_{n \in \mathbb{Z}_{+}}$which always satisfies the following conditions.

(F1) The sequence $\left\{F_{n}\right\}_{n \in \mathbb{Z}_{+}}$is stationary, with the common value of $\mathbb{P}\left(F_{n}\right)$ strictly positive.

(F2) For each $n$, the event $F_{n}$ is defined in terms of the 'future' process $\left\{\xi_{m}\right\}_{m>n}$, i.e. $\mathbf{1}_{F_{n}}=$ $g\left(\xi_{n+1}, \xi_{n+2}, \ldots\right)$ for some function $g$ (which by stationarity is independent of $n$ ). 
Here the future dependence of each of the events $F_{n}$ may be on either the finite or the infinite future.

It is our intention to define, in terms of the process $\left\{\xi_{n}\right\}$ and the sequence $\left\{F_{n}\right\}$, a sequence of random times $0 \leq \tau_{0}<\tau_{1}<\cdots$ on the nonnegative integers $\mathbb{Z}_{+}$. Our interest is in the behaviour of the successive segments of processes $\left\{\xi_{\tau_{k}+1}, \ldots, \xi_{\tau_{k+1}}\right\}, k \geq 0$. (Note that the definition of any such segment $\left\{\xi_{\tau_{k}+1}, \ldots, \xi_{\tau_{k+1}}\right\}$ includes a specification of its length $\tau_{k+1}-\tau_{k}$.) It is convenient to take a 'point process' approach, and to define first a further sequence of events $\left\{A_{n}\right\}_{n \in \mathbb{Z}_{+}}$; the times $\tau_{k}$ are then defined to be the successive times $n \geq 0$ such that the event $A_{n}$ occurs. (We observe that the need to unambiguously index the times $\tau_{k}$ obliges us to choose some origin 0 of time, and it is then convenient to restrict attention to behaviour subsequent to time 0. However, to the extent that the occurrence times of the events $A_{n}$ may be viewed as a point process on the positive integers, much of what follows may be extended without difficulty to the entire set $\mathbb{Z}$ of all the integers.)

Remark 1. Insofar as the occurrence times of the events $A_{n}$ may be regarded as a point process on the integers, the determination of their locations, in the results below, is the result of simultaneous, and essentially Markovian, conditioning from both the past and the future. There are therefore connections with the theory of one-dimensional Markov random fields. However, for our present purposes, it is natural to define the conditions for these results directly in terms of events. The distribution of the point process is then induced by the underlying i.i.d. driving sequence $\left\{\xi_{n}\right\}$.

Our main result of this section is now the following theorem. For the result to hold, we require some conditions which imply, in particular, that each event $A_{n}$ may be represented as an intersection $A_{n}=H_{n} \cap F_{n}$ of a 'past' event $H_{n} \in \sigma_{n}$ and the 'future' event $F_{n}$ defined earlier (see Remark 2 below).

Theorem 1. Let a sequence of events $\left\{A_{n}\right\}_{n \in \mathbb{Z}_{+}}$be given. Let $\tau_{0}=\min \left\{n \geq 0: \mathbf{1}_{A_{n}}=1\right\}$, and, for $k \geq 0$, let $\tau_{k+1}=\min \left\{n>\tau_{k}: \mathbf{1}_{A_{n}}=1\right\}$. Assume that $\tau_{k}<\infty$ almost surely (a.s.) for all $k$. Also, let the following be given: a sequence of 'future' events $\left\{F_{n}\right\}_{n \in \mathbb{Z}_{+}}$satisfying the earlier conditions (F1) and (F2), sequences $\left\{H_{n}^{\prime}\right\}_{n \in \mathbb{Z}_{+}}$and $\left\{H_{n}^{\prime \prime}\right\}_{n \in \mathbb{Z}_{+}}$of 'past' events such that, for each $n$, we have $H_{n}^{\prime} \in \sigma_{n}$ and $H_{n}^{\prime \prime} \in \sigma_{n}$, and, finally, an array of events $\left\{E_{n, n+m}\right\}_{n \geq 0, m>0}$ with each $E_{n, n+m} \in \sigma_{n+1, n+m}$ and such that, for each fixed $m$, the sequence $\left\{E_{n, n+m}\right\}_{n \geq 0}$ is stationary. Suppose further that all these sequences are linked by the following relations: for $n \geq 0$,

$$
\left\{\tau_{0}=n\right\} \equiv A_{0}^{\mathrm{c}} \cap \cdots \cap A_{n-1}^{\mathrm{c}} \cap A_{n}=H_{n}^{\prime} \cap F_{n},
$$

and, for $0 \leq n^{\prime}<n$,

$$
\left\{\text { there exists } k: \tau_{k}=n^{\prime}, \tau_{k+1}=n\right\} \equiv A_{n^{\prime}} \cap A_{n^{\prime}+1}^{\mathrm{c}} \cap \cdots \cap A_{n-1}^{\mathrm{c}} \cap A_{n}=H_{n^{\prime}}^{\prime \prime} \cap E_{n^{\prime}, n} \cap F_{n} .
$$

Then the successive segments of processes $\left\{\xi_{\tau_{k}+1}, \ldots, \xi_{\tau_{k+1}}\right\}, k \geq 0$, are i.i.d. In particular, the random variables $\tau_{k+1}-\tau_{k}, k \geq 0$, are i.i.d. Furthermore, for some constant $a>0$ and all $k \geq 0$,

$$
\mathbb{P}\left(\tau_{k+1}-\tau_{k}=n\right)=a \mathbb{P}\left(E_{0, n}\right) .
$$

Note that it follows directly from (2) and (3) that, for all $n$, we have $A_{n} \subseteq F_{n}$, and from (3) that, for all $n^{\prime}$, we have $A_{n^{\prime}} \subseteq H_{n^{\prime}}^{\prime \prime}$. Hence, we note that

$$
A_{n} \subseteq H_{n}^{\prime \prime} \cap F_{n}, \quad n \geq 0 .
$$


Proof of Theorem 1. Fix $k \geq 0$. For any $0 \leq n_{0}<\cdots<n_{k+1}$, it follows, from (2), (3), and the observation that $A_{n} \subseteq F_{n}$ for all $n$, that the following representation holds:

$$
\begin{aligned}
& \left\{\tau_{0}=n_{0}, \ldots, \tau_{k}=n_{k}, \tau_{k+1}=n_{k+1}\right\} \\
& \quad=H_{n_{0}}^{\prime} \cap H_{n_{0}}^{\prime \prime} \cap E_{n_{0}, n_{1}} \cap H_{n_{1}}^{\prime \prime} \cap \cdots \cap H_{n_{k}}^{\prime \prime} \cap E_{n_{k}, n_{k+1}} \cap F_{n_{k+1}} .
\end{aligned}
$$

Here the intersection of all but the last two events in (5), say $\widetilde{H}_{n_{k}}$, belongs to the $\sigma$-algebra $\sigma_{n_{k}}$ and is the same for all values of $n_{k+1}$. Thus, for any events $G_{n_{k}} \in \sigma_{n_{k}}$ and $G_{n_{k}, n_{k+1}} \in \sigma_{n_{k}+1, n_{k+1}}$,

$$
\begin{aligned}
\mathbb{P}\left(\left\{\tau_{0}\right.\right. & \left.\left.=n_{0}, \ldots, \tau_{k}=n_{k}, \tau_{k+1}=n_{k+1}\right\} \cap G_{n_{k}} \cap G_{n_{k}, n_{k+1}}\right) \\
& =\mathbb{P}\left(G_{n_{k}}^{\prime} \cap E_{n_{k}, n_{k+1}} \cap G_{n_{k}, n_{k+1}} \cap F_{n_{k+1}}\right) \\
& =\mathbb{P}\left(G_{n_{k}}^{\prime}\right) \mathbb{P}\left(E_{n_{k}, n_{k+1}} \cap G_{n_{k}, n_{k+1}}\right) \mathbb{P}\left(F_{n_{k+1}}\right),
\end{aligned}
$$

where the event $G_{n_{k}}^{\prime}=\widetilde{H}_{n_{k}} \cap G_{n_{k}}$ belongs to $\sigma_{n_{k}}$ and is the same for all values of $n_{k+1}$ and all events $G_{n_{k}, n_{k+1}}$. Thus, also using the stationarity of the sequence $\left\{F_{n}\right\}_{n \in \mathbb{Z}_{+}}$and (for each $m$ ) of the sequence $\left\{E_{n, n+m}\right\}_{n \geq 0}$, it follows that, conditional on $\left\{\tau_{0}=n_{0}, \ldots, \tau_{k}=n_{k}\right\}$ and the process $\left\{\xi_{n}\right\}_{1 \leq n \leq n_{k}}$, the distribution of $\left\{\xi_{\tau_{k}+1}, \ldots, \xi_{\tau_{k+1}}\right\}$ is that of $\left\{\xi_{\tau_{0}+1}, \ldots, \xi_{\tau_{1}}\right\}$ conditional on the occurrence of the event $\left\{\tau_{0}=n\right\}$ for any $n$ such that the latter probability is strictly positive. All the assertions of the theorem now follow. In particular, to establish (4), we consider (6) again with $G_{n_{k}}=G_{n_{k}, n_{k+1}}=\Omega$ and with $n_{k+1}=n_{k}+n$, where $n$ is fixed. Then we sum expression (6) over all $0 \leq n_{0}<\cdots<n_{k}$ to obtain $\mathbb{P}\left(\tau_{k+1}-\tau_{k}=n\right)$. This is of the form $a \mathbb{P}\left(E_{0, n}\right)$, where, clearly, $a$ does not depend on $n$; therefore, $a$ also does not depend on $k$ since the probabilities $a \mathbb{P}\left(E_{0, n}\right)$ sum to 1 .

Remark 2. It is worth pausing to note, in somewhat intuitive terms, the significance of the conditions of Theorem 1. Note first that it follows straightforwardly from (2) and (3) that, for all $n$, we have

$$
A_{n}=H_{n} \cap F_{n} \quad \text { with } \quad H_{n}=H_{n}^{\prime} \cup \bigcup_{0 \leq n^{\prime}<n} H_{n^{\prime}}^{\prime \prime} \cap E_{n^{\prime}, n} \in \sigma_{n} .
$$

Suppose now that we proceed forward in time with the process $\left\{\xi_{n}\right\}$. At each time $n$ such that the event $A_{n}$ occurs, we learn something about the future evolution of the process, namely that the event $F_{n}$ occurs. In order to have some regeneration at this time, we need to ensure that, at each such time $n$, given the knowledge that $F_{n}$ occurs, our knowledge at that time about the future probabilistic behaviour of the process is not further conditioned by our knowledge of whether or not, for each earlier time $n^{\prime}<n$, the event $A_{n^{\prime}}$ occurs. This is essentially guaranteed by condition (3), which is in effect a form of monotonicity condition.

We now give some special cases of Theorem 1 . Corollary 1 below will be applied later to the two-state contact process of Section 3.1 and to the basic infinite-bin model of Section 4.1.

Corollary 1. Suppose that, for all $n$, we have $A_{n}=F_{n}$, and that the sequence $\left\{F_{n}\right\}_{n \in \mathbb{Z}_{+}}$ satisfies the earlier conditions (F1) and (F2) and, additionally, the monotonicity condition

$$
F_{n} \cap F_{n+m}=E_{n, n+m}^{\prime} \cap F_{n+m}, \quad n \geq 0, m>0,
$$

for some array of events $\left\{E_{n, n+m}^{\prime}\right\}_{n \geq 0, m>0}$ with each $E_{n, n+m}^{\prime} \in \sigma_{n+1, n+m}$ and such that, for each fixed $m$, the sequence $\left\{E_{n, n+m}^{\prime}\right\}_{n \geq 0}$ is stationary. Then the conclusions of Theorem 1 follow (with (4) holding for appropriately defined $E_{0, n}$ ).

Proof. Note first that (7) also implies that

$$
F_{n}^{\mathrm{c}} \cap F_{n+m}=\left(E_{n, n+m}^{\prime}\right)^{\mathrm{c}} \cap F_{n+m}, \quad n \geq 0, m>0 .
$$


Since $A_{n}=F_{n}$ for all $n$, it follows from (7) and (8) that, for all $n \geq 0$,

$$
A_{0}^{\mathrm{c}} \cap \cdots \cap A_{n-1}^{\mathrm{c}} \cap A_{n}=\left(E_{0, n}^{\prime}\right)^{\mathrm{c}} \cap \cdots \cap\left(E_{n-1, n}^{\prime}\right)^{\mathrm{c}} \cap F_{n}
$$

and, for $0 \leq n^{\prime}<n$,

$$
A_{n^{\prime}} \cap A_{n^{\prime}+1}^{\mathrm{c}} \cap \cdots \cap A_{n-1}^{\mathrm{c}} \cap A_{n}=E_{n^{\prime}, n}^{\prime} \cap\left(E_{n^{\prime}+1, n}^{\prime}\right)^{\mathrm{c}} \cap \cdots \cap\left(E_{n-1, n}^{\prime}\right)^{\mathrm{c}} \cap F_{n} .
$$

Thus, the conditions of Theorem 1 are readily seen to be satisfied with $\left\{F_{n}\right\}_{n \in \mathbb{Z}_{+}}$as here, and for appropriately defined $\left\{H_{n}^{\prime}\right\}_{n \in \mathbb{Z}_{+}},\left\{H_{n}^{\prime \prime}\right\}_{n \in \mathbb{Z}_{+}}$(with $H_{n}^{\prime \prime}=\Omega$ for all $n$ ), and $\left\{E_{n, n+m}\right\}_{n \geq 0, m>0}$.

Remark 3. We can now provide some explanation for the observations of Example 1 in the introduction. In the variant (a), condition (7) of Corollary 1 is readily seen to be satisfied, with $E_{n, n+m}^{\prime}=\left\{\sum_{i=1}^{m^{\prime}} \xi_{n+i} \geq 0\right.$ for all $\left.1 \leq m^{\prime} \leq m\right\}$. In the variant (b) of Example 1 the monotonicity condition (7) of Corollary 1 (with the sequence $\left\{F_{n}\right\}_{n \geq 0}$ replaced by $\left\{F_{n}^{\prime}\right\}_{n \geq 0}$ ) clearly cannot be satisfied, and, more generally, the conditions of Theorem 1 cannot be satisfied (for otherwise that theorem would contradict the known behaviour for this example). However, for the variant (c) of Example 1, we may observe that condition (7) of Corollary 1 (again with the sequence $\left\{F_{n}\right\}_{n \geq 0}$ replaced by $\left\{F_{n}^{\prime}\right\}_{n \geq 0}$ ) is satisfied whenever $m \geq 2$, with $E_{n, n+m}^{\prime}=$ $\left\{\sum_{i=1}^{m^{\prime}} \xi_{n+i} \geq 0\right.$ for all $1 \leq m^{\prime} \leq m$ and $\left.\xi_{n+2}=0\right\}$. Since the enforced minimum separation $\tau_{k}-\tau_{k-1} \geq 2$ for $k \geq 1$ implies that $A_{n} \cap A_{n+1}=\varnothing$ for all $n$, this restricted version of condition (7), coupled with the proof of Corollary 1 , is now sufficient to establish that the conditions of Theorem 1 are satisfied as before.

We now give a generalisation of Corollary 1 in which the sequence $\left\{A_{n}\right\}_{n \in \mathbb{Z}_{+}}$is defined by $A_{n}=H_{n} \cap F_{n}$ for the sequence $\left\{F_{n}\right\}_{n \in \mathbb{Z}_{+}}$as above and with $\left\{H_{n}\right\}_{n \in \mathbb{Z}_{+}}$some sequence such that each $H_{n} \in \sigma_{n}$. Here a monotonicity condition is additionally required on the sequence $\left\{H_{n}\right\}_{n \in \mathbb{Z}_{+}}$. However, the monotonicity condition on the sequence $\left\{F_{n}\right\}_{n \in \mathbb{Z}_{+}}$is only required to hold in relation to those times $n$ at which the event $H_{n}$ occurs. The result, which reduces to Corollary 1 in the case where $H_{n}=\Omega$ for all $n$, is entirely natural for many applications. It will be applied in Example 2 below, to the three-state contact process of Section 3.2, and to the continuous-space 'infinite-bin' model of Section 4.2.

Corollary 2. Suppose that, for all $n$, we have $A_{n}=H_{n} \cap F_{n}$, where the sequence $\left\{F_{n}\right\}_{n \in \mathbb{Z}_{+}}$ satisfies the earlier conditions (F1) and (F2) and the sequence $\left\{H_{n}\right\}_{n \in \mathbb{Z}_{+}}$is such that $H_{n} \in \sigma_{n}$ for all $n$. Suppose further that these sequences satisfy the following monotonicity conditions.

(a) For all $n \geq 0$ and $m>0$,

$$
A_{n} \cap A_{n+m}=H_{n} \cap E_{n, n+m}^{\prime} \cap A_{n+m},
$$

where the array of events $\left\{E_{n, n+m}^{\prime}\right\}_{n \geq 0, m>0}$ is such that each $E_{n, n+m}^{\prime} \in \sigma_{n+1, n+m}$ and, for each fixed $m$, the sequence $\left\{E_{n, n+m}^{\prime}\right\}_{n \geq 0}$ is stationary.

(b) For all $n \geq 0$ and $m>0$,

$$
A_{n} \cap H_{n+m}=A_{n} \cap E_{n, n+m}^{\prime \prime},
$$

where the array of events $\left\{E_{n, n+m}^{\prime \prime}\right\}_{n \geq 0, m>0}$ is again such that each $E_{n, n+m}^{\prime \prime} \in \sigma_{n+1, n+m}$ and, for each fixed $m$, the sequence $\left\{E_{n, n+m}^{\prime \prime}\right\}_{n \geq 0}$ is stationary.

Then the conclusions of Theorem 1 follow (again with (4) holding for appropriately defined $\left.E_{0, n}\right)$. 
Proof. The proof is similar to that of Corollary 1, if a little messier. It is necessary to verify conditions (2) and (3) of Theorem 1, in which the sequence $\left\{F_{n}\right\}_{n \in \mathbb{Z}_{+}}$of that theorem is as given here. Note first that (9) also implies that, for all $n \geq 0$ and $m>0$,

$$
H_{n} \cap F_{n}^{\mathrm{c}} \cap A_{n+m}=H_{n} \cap\left(E_{n, n+m}^{\prime}\right)^{\mathrm{c}} \cap A_{n+m},
$$

while (10) similarly also implies that, for all $n \geq 0$ and $m>0$,

$$
A_{n} \cap H_{n+m}^{\mathrm{c}}=A_{n} \cap\left(E_{n, n+m}^{\prime \prime}\right)^{\mathrm{c}} .
$$

Consider first the verification of condition (3). For $0 \leq n^{\prime}<n$, the event

$$
A_{n^{\prime}} \cap A_{n^{\prime}+1}^{\mathrm{c}} \cap \cdots \cap A_{n-1}^{\mathrm{c}} \cap A_{n}
$$

may be written as a union of events of the form

$$
A_{n^{\prime}} \cap B_{n^{\prime}+1} \cap \cdots \cap B_{n-1} \cap A_{n},
$$

where, for each $n^{\prime}<k<n$, the event $B_{k}$ is either $H_{k}^{\mathrm{c}}$ or $H_{k} \cap F_{k}^{\mathrm{c}}$. Now using (12) to simplify $A_{n^{\prime}} \cap H_{k}^{\mathrm{c}}$, (11) and then (12) to simplify $H_{k} \cap F_{k}^{\mathrm{c}} \cap A_{n}$, and, finally, (9) to simplify $A_{n^{\prime}} \cap A_{n}$, it follows that each of the events given by (14), and so also the event given by (13), has a representation as $H_{n^{\prime}} \cap E_{n^{\prime}, n} \cap F_{n}$, where, by construction, the array $\left\{E_{n^{\prime}, n}\right\}_{n^{\prime} \geq 0, n>n^{\prime}}$ satisfies the conditions of Theorem 1 .

The verification of condition (2) of Theorem 1 (for some readily calculable sequence $\left\{H_{n}^{\prime}\right\}_{n \in \mathbb{Z}_{+}}$with each $\left.H_{n}^{\prime} \in \sigma_{n}\right)$ is similar, but simpler.

We now consider a process $\left\{X_{n}\right\}_{n \geq 0}$ which, as indicated at the beginning of this section, is adapted with respect to the filtration $\left\{\sigma_{n}\right\}_{n \in \mathbb{Z}}$ (or $\left\{\sigma_{n}\right\}_{n \in \mathbb{Z}_{+}}$). We further assume that this process is defined via the specification of $X_{0}$ and the stochastic recursion (1). This is a standard situation (but not the only one) in which the regenerative structure of the successive blocks of the process $\left\{\xi_{n}\right\}$, as identified in Theorem 1, may be inherited by a (functional of the) process $\left\{X_{n}\right\}$. We introduce here a number of typical scenarios which will be complemented by the examples of the following sections.

In what follows we wish to consider, for any time $n$, the dynamics of the sequence $\left\{X_{n+i}\right\}_{i \geq 0}$, and of functionals of this sequence, relative to 'an initial' $X_{n}$. (One may think of a growth model in which we are adding points at each time, or of a model in which we centre the system around the value of $X_{n}$ at time $n$.) In order to do this, we introduce functions $R_{i}\left(X_{n}, X_{n+i}\right), i \geq 1$, which capture this relative behaviour. We assume further that each such function $R_{i}$ acts as $R_{i}: X^{2} \rightarrow \mathcal{Y}$, where $\left(\mathcal{X}, \mathcal{B}_{X}\right)$ is the space in which $X_{n}$ take values and $\left(\mathcal{Y}, \mathcal{B}_{y}\right)$ is another measurable space. In various of the remaining examples of this paper, we indicate precisely the form of the functions $R_{i}$.

Recall that a sequence, say $\left\{Y_{n}\right\}$, is stationary one-dependent if it is stationary and, for any $n$, the families of random variables $\left\{Y_{k}, k<n\right\}$ and $\left\{Y_{k}, k>n\right\}$ are independent. The following result is an immediate extension of Theorem 1 and the given conditions (15), (16), and (17).

Theorem 2. Suppose again that the sequence $\left\{\xi_{n}\right\}_{n \in \mathbb{Z}}$ consists of i.i.d. random variables and that the random times $0 \leq \tau_{0}<\tau_{1}<\cdots$ are defined as in Theorem 1 , with all the conditions of that theorem holding.

(a) Suppose that the functions $R_{i}, i \geq 1$, introduced above are such that, for any $n$, given that the event $A_{n}$ occurs, each random variable $R_{i}\left(X_{n+i}, X_{n}\right)$ is a measurable function 
of $\xi_{n+1}, \ldots, \xi_{n+i}$ only, i.e. for every $i \geq 1$,

$$
R_{i}\left(X_{n+i}, X_{n}\right) \mathbf{1}_{A_{n}}=g_{i}\left(\xi_{n+1}, \ldots, \xi_{n+i}\right) \mathbf{1}_{A_{n}} .
$$

Then the random elements

$$
\left(R_{i}\left(X_{\tau_{j}+i}, X_{\tau_{j}}\right), i=1, \ldots, \tau_{j+1}-\tau_{j}\right)
$$

are i.i.d. in $j$.

(b) Suppose, more generally, that, for some fixed $m \geq 1$, the functions $R_{i}, i \geq 1$, introduced above are now as follows: for any $n$, given that the event $A_{n}$ occurs, each random variable $R_{i}\left(X_{n+i}, X_{n}\right)$ is a measurable function of $\xi_{n-m+1}, \ldots, \xi_{n+i}$ only, i.e. for every $i \geq 1$,

$$
R_{i}\left(X_{n+i}, X_{n}\right) \mathbf{1}_{A_{n}}=g_{i}\left(\xi_{n-m+1}, \ldots, \xi_{n+i}\right) \mathbf{1}_{A_{n}}
$$

and that

$$
\tau_{n+1}-\tau_{n} \geq m \text { a.s. for all } n .
$$

Then the random elements (16) are stationary one-dependent.

Recall that a random sequence $\left\{Z_{n}\right\}$ is regenerative if there exist (random) times $0 \leq \tau_{0}<$ $\tau_{1}<\cdots$ such that the random elements

$$
\begin{aligned}
& Y_{0}:=\left(\tau_{0} ; Z_{0}, \ldots, Z_{\tau_{0}}\right), \\
& Y_{1}:=\left(\tau_{1}-\tau_{0} ; Z_{\tau_{0}+1}, \ldots, Z_{\tau_{1}}\right), \\
& Y_{2}:=\left(\tau_{2}-\tau_{1} ; Z_{\tau_{1}+1}, \ldots, Z_{\tau_{2}}\right),
\end{aligned}
$$

etc. are mutually independent and the elements $\left\{Y_{k}\right\}_{k \geq 1}$ are identically distributed. The random sequence $\left\{Z_{n}\right\}$ is wide-sense regenerative if, for each $n \geq 0$, the distribution of the sequence $\left\{Z_{\tau_{n}+k}, k \geq 0\right\}$ does not depend on $\tau_{n}$. Furthermore, $\left\{Z_{n}\right\}$ possesses one-dependent regenerative cycles induced by $\left\{\tau_{n}\right\}$ if the sequence $\left\{Y_{k}\right\}_{k \geq 0}$ is one-dependent, and the random variables $\left\{Y_{k}\right\}_{k \geq 1}$ are identically distributed. The latter two properties imply that the sequence $\left\{Y_{k}\right\}_{k \geq 1}$ is stationary and that the time instants $\left\{\tau_{k}\right\}$ form a renewal process.

Corollary 3. Suppose that the sequence $\left\{\xi_{n}\right\}_{n \in \mathbb{Z}}$ consists of i.i.d. random variables and that the random times $0 \leq \tau_{0}<\tau_{1}<\cdots$ are defined as in Theorem 1, with all the conditions of that theorem holding. Suppose that the functions $R_{i}, i \geq 1$, introduced above are such that either the conditions of part (a) or those of part (b) of Theorem 2 hold. Finally, suppose that the (common) distribution of the random variables $\tau_{n}-\tau_{n-1}, n \geq 1$, is aperiodic, i.e. $\operatorname{GCD}\left\{j: \mathbb{P}\left(\tau_{1}-\tau_{0}=j\right)>0\right\}=1$.

For any $n>\tau_{0}$, let

$$
Z_{n}=R_{n-\tau_{j}}\left(X_{n}, X_{\tau_{j}}\right) \quad \text { if } \tau_{j}<n \leq \tau_{j+1}
$$

Then the sequence $Z_{n}$ converges in the total variation norm to a proper limiting random variable.

Indeed, by Theorem 1 , the sequence $Z_{n}$ is wide-sense regenerative and, by Theorem 2 , it possesses one-dependent regenerative cycles, so that Corollary 3 follows from the stability theorem for wide-sense regenerative processes; see, e.g. Section 10 of [27] or [1]. 
Example 2. (Random walk with positive drift.) We extend Example 1 to consider a general random walk with positive drift. Such a process provides possibly the simplest instance of the application of the above theory, and our aims here are to both demonstrate the use of Theorem 1 and illustrate the use of conditioning simultaneously on both past and future events. The results we give for this example are not new, but rather illustrate the immediate applicability of the present theory. The sequence $\left\{\xi_{n}\right\}_{n \geq 1}$ consists as usual of i.i.d. nondegenerate random variables $\xi_{n}$ with positive mean $a=\mathbb{E} \xi>0$. To make the example nontrivial, assume that $\mathbb{P}(\xi<0)>0$. The process of interest is the random walk $\left\{S_{n}\right\}_{n \geq 0}$ with $S_{0}=0$ and $S_{n}=\sum_{i=1}^{n} \xi_{i}$ (here we use the notation $S_{n}$ instead of $X_{n}$ ).

We define the earlier sequence $\left\{F_{n}\right\}_{n \in \mathbb{Z}_{+}}$, satisfying conditions (F1) and (F2), by taking each $F_{n}$ to be the event that $\sum_{k=1}^{m} \xi_{n+k}>0$ for all $m \geq 1$, i.e. that $S_{m}>S_{n}$ for all $m>n$.

First, for a trivial application, we define the events $A_{n}$ of Theorem 1 by, for each $n, A_{n}=F_{n}$, so that the random times $\tau_{k}, k \geq 0$, are simply the successive occurrence times of the events $F_{n}$, and are simply the last exit times of the process $\left\{S_{n}\right\}$ above successive levels. As in the variant (a) of the earlier Example 1, condition (7) of Corollary 1 is easily seen to be satisfied (with $E_{n, n+m}^{\prime}=\left\{\sum_{k=1}^{m^{\prime}} \xi_{n+k}>0\right.$ for all $\left.1 \leq m^{\prime} \leq m\right\}$ ). Thus, the conclusions of Theorem 1 follow, and we have the well-known and elementary result that the segments of the process $\left\{S_{n}\right\}$ between the successive last exit times above are i.i.d.

We also observe that the functionals $R_{i}$ defined above are typically given by $R_{i}\left(S_{n+i}, S_{n}\right)=$ $S_{n+i}-S_{n}$.

A more interesting application is given by defining the events $A_{n}$ of Theorem 1 by, for each $n$, $A_{n}=H_{n} \cap F_{n}$, where $H_{n}$ is the event that $S_{n^{\prime}}<S_{n}$ for all $0 \leq n^{\prime}<n$. Thus, the times $\tau_{k}$ are the successive times $n$ at which both $S_{n^{\prime}}<S_{n}$ for all $n^{\prime}<n$ and $S_{n^{\prime}}>S_{n}$ for all $n^{\prime}>n$. The sequences of events $\left\{F_{n}\right\}_{n \in \mathbb{Z}_{+}}$and $\left\{H_{n}\right\}_{n \in \mathbb{Z}_{+}}$satisfy conditions (9) and (10) of Corollary 2, with $E_{n, n+m}^{\prime}$ as above and $E_{n, n+m}^{\prime \prime}=\left\{S_{n^{\prime}}<S_{n+m}\right.$ for all $\left.n \leq n^{\prime}<n+m\right\}$. Hence, again, the conclusions of Theorem 1 follow, and we again have the result that the segments of the process $\left\{S_{n}\right\}$ between the successive times $\tau_{k}$ are i.i.d. A weak consequence is that these times themselves form a delayed renewal process.

Here, again, the functionals $R_{i}$ of Section 2 are typically given by $R_{i}\left(S_{n+i}, S_{n}\right)=S_{n+i}-S_{n}$.

\section{The asymptotic behaviour of supercritical contact processes}

The theory of Section 2 has applications to a variety of particle systems and oriented percolation models. In this section we consider some fairly general discrete-time contact, or oriented percolation, processes on the integers $\mathbb{Z}$. These models are normally studied in a continuous-time setting, and it is clear that the relevant theory of Section 2 could, at the cost of some work, be adapted to that setting.

In Section 3.1 we study a modest generalisation of the traditional two-state contact process on $\mathbb{Z}$, in which, at each time $n$, each site $a \in \mathbb{Z}$ is either healthy (state 0 ) or infected (state 1 ). We use our earlier theory to study the behaviour of the right-endpoint process, defined for each time $n$ to be the rightmost infected site at time $n$. The underlying ideas here are those of Kuczek [19] and of Mountford and Sweet [23] — the somewhat greater generality of the model considered here makes little difference. However, we show that the results of Kuczek are an almost immediate application of the theory of Section 2, and become clearer when thus understood. (These ideas are further used by Mountford and Sweet, but the main work of their paper is an additional 'block' construction to show that a certain event has a strictly positive probability - see the discussion at the end of this section.) The basic theory of Section 3.1 is further a necessary preliminary for applications to other particle system models. We give one such application in 
Section 3.2 in which we study an extension to a three-state process. In this model, which has been considered by a number of authors, healthy sites differ in their susceptibility to subsequent infection according to whether they have previously been infected. Tzioufas [28] deduced rightendpoint behaviour for the 'reverse-immunisation' version of the process, in which previously infected sites are easier to reinfect. His argument used a monotonicity property which fails to hold in the 'immunisation' version of the process, in which previously infected sites are more difficult to reinfect. We show how this difficulty is overcome by a suitable definition of the sets $F_{n}$ of Section 2.

\subsection{The two-state contact process}

Consider a process in which sites, indexed by the integers $\mathbb{Z}$, are at each time $n$ either healthy or infected. We define the state $X_{n}$ of the process at time $n$ to be the set of infected sites at that time. Between times $n$ and $n+1$ each site $x \in X_{n}$ which is infected at time $n$ produces a set $\eta_{n+1, x} \subseteq \mathbb{Z}$ of descendants, which is again a subset of $\mathbb{Z}$; at time $n+1$ these descendants infect a set of sites $x+\eta_{n+1, x} \subseteq \mathbb{Z}$, where, for any $x \in \mathbb{Z}$ and any $A \subseteq \mathbb{Z}$, we define $x+A=\{x+a: a \in A\}$. The state $X_{n+1}$ of the process at time $n+1$ is given by

$$
X_{n+1}=\bigcup_{x \in X_{n}}\left(x+\eta_{n+1, x}\right),
$$

i.e. the union over $x \in X_{n}$ of the sites infected by the descendants of these $x$. Finally, we assume that the random sets $\eta_{n+1, x}$ are i.i.d. over both times $n$ and sites $x$. This model is a fairly general form of the discrete-time version of the one-dimensional contact process, or oriented percolation. Note that we do not make the restriction (common for both discrete-time oriented percolation and continuous-time contact processes) that, for each $n$ and $x$, the random set $\eta_{n+1, x}$ is such that the events $\left\{a \in \eta_{n+1, x}\right\}$ are independent over $a$.

Let $p$ be the probability that the process started with $X_{0}=\{0\}$, say, survives, i.e. $X_{n} \neq \varnothing$ for all $n \geq 0$. Suppose that $p>0$, so that the process is described as supercritical. Our interest is then in the long-run behaviour of this process. In particular, we are concerned with the behaviour of the right-endpoint process $\left\{r_{n}\right\}_{n \geq 0}$, conditional on survival, where we define $r_{n}=\max \left(x: x \in X_{n}\right)$ (with $r_{n}=-\infty$ in the case where $X_{n}$ is empty). This, coupled with the behaviour of the corresponding left-endpoint process, determines the growth rate of the process. We assume that $X_{0}$ is such that $r_{0}<\infty$ (and, hence, $r_{n}<\infty$ for all $n$ ); usually, $r_{0}=0$.

Consider first the case in which the process possesses the following skip-free property: for all $n$ and all $x, y \in \mathbb{Z}$,

$$
x<y \quad \Longrightarrow \quad x+a \leq y+b \quad \text { for all } a \in \eta_{n+1, x}, b \in \eta_{n+1, y} \text { a.s. }
$$

This is the discrete-time version of the nearest-neighbour property of the contact process on $\mathbb{Z}$. The process $\left\{r_{n}\right\}_{n \geq 0}$ was studied by Galves and Presutti [17] and by Kuczek [19]. The argument here is essentially a rephrasing, in the framework of the present paper, of that of Kuczek, and is given not only as an example and for completeness, but because it is required for subsequent developments, in particular for the theory of Section 3.2, in which the present arguments are extended and generalised.

We take the driving sequence $\left\{\xi_{n}\right\}_{n \geq 1}$ (the index range $n \geq 1$ is sufficient), introduced in Section 2, to be defined as follows. For each $n, \xi_{n}=\left\{\xi_{n, z}\right\}_{z \leq 0}$, where, for each nonpositive integer $z, \xi_{n, z}$ has the same distribution as any of the random sets $\eta_{n, x}$ identified above. 
In addition to being identically distributed, the random sets $\xi_{n, z}$ are taken to be independent over all $n$ and all $z$. We now define the process $\left\{X_{n}\right\}_{n \geq 0}$ via a stochastic recursion

$$
X_{n+1}=f\left(X_{n}, \xi_{n+1}\right)
$$

for each $x \in X_{n}$, the random set $\eta_{n+1, x}$ of its descendants at time $n+1$ is given by

$$
\eta_{n+1, x}=\xi_{n+1, x-r_{n}}
$$

Thus, in particular - and this is critical for the understanding of the argument below, in which everything is in effect viewed from the right endpoints of the processes of interest - the random set $\xi_{n+1,0}$ determines the set of descendants of the rightmost infected site $r_{n}$ at time $n$, and, for every other infected site at time $n$, we count its distance from $r_{n}$ in order to determine which of the random sets $\xi_{n+1, z}$ to use for its set of descendants. Different initial sets $X_{0}$ of infected sites lead to different instances of the process $\left\{X_{n}\right\}_{n \geq 0}$.

For each $n \geq 0$, consider the process $\left\{X_{n^{\prime}}^{(n)}\right\}_{n^{\prime} \geq n}$ defined by $X_{n}^{(n)}=\{0\}$ and $X_{n^{\prime}+1}^{(n)}=$ $f\left(X_{n^{\prime}}^{(n)}, \xi_{n^{\prime}+1}\right)$ for $n^{\prime} \geq n$. Define also the associated right-endpoint process $\left\{r_{n^{\prime}}^{(n)}\right\}_{n^{\prime} \geq n}^{n^{\prime}+1}$ by $r_{n^{\prime}}^{(n)}=\max \left(x: x \in X_{n^{\prime}}^{(n)}\right)$ (again with $r_{n^{\prime}}^{(n)}=-\infty$ in the case where $X_{n^{\prime}}^{(n)}$ is empty).

We define the sequence of events $\left\{F_{n}\right\}_{n \in \mathbb{Z}_{+}}$of Section 2 saying that the event $F_{n}$ occurs if and only if the process $\left\{X_{n^{\prime}}^{(n)}\right\}_{n^{\prime} \geq n}$ survives for all future time. It follows from the definition of the process $\left\{X_{n^{\prime}}^{(n)}\right\}_{n^{\prime} \geq n}$ that the sequence $\left\{F_{n}\right\}_{n \in \mathbb{Z}_{+}}$satisfies conditions (F1) and (F2) of Section 2 . In particular, the common value of the probability of the events $F_{n}$ is $p$, which, by our earlier assumption of supercriticality is strictly positive.

We define the events $A_{n}$ of Theorem 1 by, for each $n, A_{n}=F_{n}$, so that the random times $\tau_{k}, k \geq 0$, are simply the successive occurrence times of the events $F_{n}$. It is our intention to apply Corollary 1. Fix therefore $n \geq 0$ and $m>0$, and suppose that the event $F_{m+n}$ occurs. Then if the process $\left\{X_{n^{\prime}}^{(n)}\right\}_{n^{\prime} \geq n}$ also survives to time $n+m$, i.e. $X_{n+m}^{(n)} \neq \varnothing$, it follows from (19) and (20) that

$$
r_{n+m+n^{\prime}}^{(n)}=r_{n+m}^{(n)}+r_{n+m+n^{\prime}}^{(n+m)} \text { for all } n^{\prime} \geq 0 .
$$

It now follows that, given that the event $F_{m+n}$ occurs, the event $F_{n}$ occurs if and only if there occurs the event $E_{n, n+m}^{\prime}$ that the process $\left\{X_{n^{\prime}}^{(n)}\right\}_{n^{\prime} \geq n}$ survives to time $n+m$. Thus, the monotonicity condition (7) of Corollary 1 is satisfied (with $F_{n}, F_{n+m}$, and $E_{n, n+m}^{\prime}$ as defined here) and, by construction, the array $\left\{E_{n, n+m}^{\prime}\right\}_{n \geq 0, m>0}$ satisfies the conditions of that corollary, so that the conclusions of Theorem 1 follow. Since the events $F_{n}$ also have strictly positive probability $p$, and since their indicator random variables $\mathbf{1}_{F_{n}}$ form a stationary ergodic sequence, the first statement of Proposition 1 below is now immediate from Theorem 1 combined with the strong law of large numbers for such sequences.

The proof of the second statement of Proposition 1 is also essentially due to Kuczek, but, as we require essentially the same argument (with a little extra complication) in Section 3.2 below, we summarise it here. Define the random time $\tau^{\prime}=\left\{\min n \geq 1: F_{n}\right.$ occurs $\}$. The common distribution of the intervals $\tau_{k+1}-\tau_{k}, k \geq 0$, is that of the random time $\tau_{1}$ conditioned on the event $\left\{\tau_{0}=0\right\}$, i.e. on the event $F_{0}$, which has strictly positive probability. The latter distribution is also that of the random time $\tau^{\prime}$ conditioned on the event $F_{0}$. Hence, for the second statement of Proposition 1, it is sufficient to show that the (unconditional) distribution of $\tau^{\prime}$ is geometrically bounded. We show that this follows from the well-known property of supercritical contact processes that if $\rho=\min \left\{n \geq 1: X_{n}^{(0)}=\varnothing\right\}$ then there exists $\alpha>0$ such that

$$
\mathbb{P}(n \leq \rho<\infty) \leq \mathrm{e}^{-\alpha n}, \quad n \geq 1 .
$$


To see that $\tau^{\prime}$ is geometrically bounded, we may proceed forward in time, starting at time 1 , checking at that and at selected subsequent times $n$ whether the event $F_{n}$ occurs: if, at any such time, it fails to do so, we wait until the process $\left\{X_{n^{\prime}}^{(n)}\right\}_{n^{\prime} \geq n}$ dies before resuming checking at subsequent times, thereby ensuring that checks are independently successful, each with probability $p>0$; the time to the occurrence of a first success, and, hence, to the occurrence of some event $F_{n}$, is thus a geometric sum of i.i.d. geometrically bounded random variables, and is hence itself geometrically bounded, implying the same result for $\tau^{\prime}$. We thus have the following proposition.

Proposition 1. The successive (segments of) processes $\left\{\xi_{\tau_{k}+1}, \ldots, \xi_{\tau_{k+1}}\right\}, k \geq 0$, are i.i.d., each with finite mean length $1 / \mathrm{p}$. Furthermore, the distribution of each of these lengths is light tailed, i.e. geometrically bounded, and in particular possesses moments of all orders.

Now let $\left\{X_{n}\right\}_{n \geq 0}$ be any version of the contact process defined by (19) such that $X_{0} \neq \varnothing$ and, if $\left\{r_{n}\right\}_{n \geq 0}$ is its right-endpoint process then $r_{0}<\infty$. Let $F$ be the event that the process $\left\{X_{n}\right\}_{n \geq 0}$ survives. Then, as in the argument above used to establish (7), the event $F$ occurs if and only if the process survives to time $\tau_{0}$. Note also that $F_{0} \subseteq F$; in the extreme case where there is a single infected site at time 0 we have $F_{0}=F$, while in the case the number of infected sites at time 0 is infinite, we have $\mathbb{P}(F)=1$. Furthermore, from the construction of the processes involved and recalling (21), conditional on the event $F$ and for all $k \geq 0$,

$$
r_{\tau_{k}+n^{\prime}}=r_{\tau_{0}}+\sum_{j=0}^{k-1} r_{\tau_{j+1}}^{\left(\tau_{j}\right)}+r_{\tau_{k}+n^{\prime}}^{\left(\tau_{k}\right)} \quad \text { for all } n^{\prime} \geq 0 .
$$

We thus immediately have the following corollary to Proposition 1.

Corollary 4. (Kuczek [19].) On the set $F$ the successive (segments of) processes $\left\{r_{\tau_{k}+1}, \ldots\right.$, $\left.r_{\tau_{k+1}}\right\}, k \geq 0$, are i.i.d. Furthermore, for some constant $\mu$,

$$
\frac{r_{n}}{n} \rightarrow \mu \quad \text { a.s. as } n \rightarrow \infty
$$

and, in the Skorokhod topology,

$$
\frac{r_{[n t]}-n t \mu}{\sqrt{n}} \rightarrow B(t) \text { in distribution as } n \rightarrow \infty,
$$

where, for any $a>0$, we denote by $[a]$ the integer part of $a$, and where $B$ is Brownian motion with some nontrivial diffusion constant.

Remark 4. We believe that it is also worth discussing briefly the more general case, considered by Mountford and Sweet [23], in which the skip-free condition (18) is replaced by the more general condition that the sets $\eta_{n+1, x}$ have bounded support, if only as an illustration of our general thesis that everything depends of the appropriate definition of the sequence $\left\{F_{n}\right\}_{n \in \mathbb{Z}_{+}}$ of Section 2. Here it is sufficient to redefine the events $F_{n}$ and to show that their common probability remains strictly positive. Thus, for each $n$, define not only the process $\left\{X_{n^{\prime}}^{(n)}\right\}_{n^{\prime} \geq n}$ as above (in which $X_{n}^{(n)}=\{0\}$ ) and its associated right-endpoint process $\left\{r_{n^{\prime}}^{(n)}\right\}_{n^{\prime} \geq n}$, but also the process $\left\{\bar{X}_{n^{\prime}}^{(n)}\right\}_{n^{\prime} \geq n}$ given by $\bar{X}_{n}^{(n)}=\mathbb{Z}_{-}$(where $\mathbb{Z}_{-}$is the set of nonpositive integers) and $\bar{X}_{n^{\prime}+1}^{(n)}=f\left(\bar{X}_{n}^{(n)}, \xi_{n+1}\right)$ for $n^{\prime} \geq n$; denote also the latter process's associated right-endpoint process by $\left\{\bar{r}_{n^{\prime}}^{(n)}\right\}_{n^{\prime} \geq n}$. Note that the process $\left\{\bar{X}_{n^{\prime}}^{(n)}\right\}_{n^{\prime} \geq n}$ survives a.s., and that, since $X_{n}^{(n)} \subset \bar{X}_{n}^{(n)}$, we have $X_{n^{\prime}}^{(n)} \subseteq \bar{X}_{n^{\prime}}^{(n)}$ for all $n^{\prime} \geq n$. The event $F_{n}$ is now defined to occur if and only if 
$r_{n^{\prime}}^{(n)}=\bar{r}_{n^{\prime}}^{(n)}$ for all $n^{\prime} \geq n$. (The latter condition implies the survival of the process $\left\{X_{n^{\prime}}^{(n)}\right\}_{n^{\prime} \geq n}$ and is equivalent to it in the earlier skip-free case.) Furthermore, the sequence $\left\{F_{n}\right\}_{n \in \mathbb{Z}_{+}}$ continues to satisfy conditions (F1) and (F2) of Section 2, provided that we can show that the common probability $p^{\prime}$ of the events $F_{n}$ is strictly positive.

As before, we define the events $A_{n}$ of Theorem 1 by $A_{n}=F_{n}$ for all $n$, so that the times $\tau_{k}$ are once more the times of successive occurrences of the events $F_{n}$. It follows from the definition of the latter events that condition (21) continues to hold for those $n \geq 0$ and $m>0$ such that event $F_{n+m}$ occurs. Thus, the conditions of Corollary 1 hold as in the skip-free case, and indeed the entire argument of that case also holds in the present more general case, subject only to the above proviso that $\mathbb{P}\left(F_{n}\right)>0$. Thus, in this case, we once more obtain Proposition 1 (with $p$ replaced by $p^{\prime}$ ) and Corollary 4 describing the behaviour of the right-endpoint process.

That $p^{\prime}>0$ is shown by Mountford and Sweet [23] -in the most difficult part of their paper-using a block construction and under a condition on the random sets $\eta_{n+1, x}$ which limits the extent of the dependence between the events $\left\{a \in \eta_{n+1, x}\right\}$. While it seems likely that $p^{\prime}>0$ in the present slightly more general model and that this should not be too difficult to show, we do not pursue this here.

\subsection{A three-state contact process with immunisation}

We consider a model in which the susceptibility of sites to infection depends on whether they have been previously infected. As noted above, such models (in continuous time) have been considered by a number of authors (see [12], [26], and [28]). We show here how rightendpoint, and hence growth, behaviour can be deduced for a model with immunisation, in which previously infected sites are more difficult to infect than those which have not previously been infected. What is interesting here is that we do not have monotonicity of the process in the initial level of infection, in that the introduction of additional infected sites at time 0 may possibly, by premature infection and then immunisation of neighbouring sites, reduce the number of infected sites at subsequent times (see [26] for details). This is in contrast to the two-state contact process and to the 'reverse-immunisation' three-state process mentioned earlier. However, in the present model there do still exist sufficient monotonicity-preserving couplings, between instances of the process with suitably different initial states, as to enable progress to be made with a little extra care, notably in the definition below of the 'future' events $F_{n}$ of Section 2 and below. A further complication is that the events $A_{n}$ of Section 2 are no longer simply defined by $A_{n}=F_{n}$, but rather the occurrence of the event $A_{n}$ depends both on the past and future behaviours of the process $\left\{\xi_{n^{\prime}}\right\}_{n^{\prime} \geq 1}$ relative to the time $n$.

We take our argument in stages: we consider first the model, then its formulation as a stochastic recursion suitable for the application of the theory of Section 2, and then the definition of the events $F_{n}$ and the times $\tau_{k}$ of Section 2; finally, we apply the earlier theory and such additional arguments as are necessary to obtain our results.

3.2.1. The model. The varying susceptibility of sites forces a more careful identification between sites at one time period and another. We therefore focus on the following generalisation of a simple oriented percolation model, which possesses the skip-free property (18) identified in the previous section and which is the discrete-time analogue of the three-state nearestneighbour contact process with a similar immunisation property. The model corresponds to oriented percolation through time on the integers in which, given the state of the process at time $n$, each site which would potentially be infected at time $n+1$ is only actually infected with some fixed probability $q$, independently of all else, unless it has never previously been infected, in which case it is infected with probability 1 . 
The state $X_{n}$ of the process $\left\{X_{n}\right\}_{n \geq 0}$ at time $n$ is given by $X_{n}=\left\{X_{n}(x), x \in \mathbb{Z}\right\}$, where each $X_{n}(x) \in\{-1,0,1\}$, and where this has the interpretation

$$
X_{n}(x)= \begin{cases}-1 & \text { if the site } x \text { is uninfected for all } n^{\prime} \leq n, \\ 0 & \text { if the site } x \text { is uninfected at time } n \text { but has previously been infected, } \\ 1 & \text { if the site } x \text { is infected at time } n\end{cases}
$$

In order to obtain a spatially symmetric model and to maintain the above skip-free property, we make the restriction that, at each time $n$, the set of sites $x$ which possibly may be infected $\left(X_{n}(x)=1\right)$ is the set of integers $x \in \mathbb{Z}$ such that $n+x$ is even. This will follow from the specification of the dynamics of the process below, provided we require that only evenly numbered sites may be infected at time 0 . (In these dynamics, which we make precise below, a site which is infected at time $n$ reverts to state 0 at time $n+1$; a site which is in state -1 or 0 at time $n$ remains in the same state at time $n+1$ unless it becomes infected at time $n+1$, in which case its state becomes 1.) The above restriction on the locations of the infected sites at any time is of a purely technical nature and is not necessary is the (more natural) continuous-time version of the process.

We assume therefore that $X_{0}$ is such that $X_{0}(x) \neq 1$ for odd $x$. The state $X_{n+1}$ of the process is obtained from $X_{n}$ as follows: for each $n$ and each $x \in \mathbb{Z}$ such that $n+x$ is even, associate a random set $\eta_{n+1, x} \subseteq\{-1,1\}$; the random sets $\eta_{n+1, x}$ are assumed i.i.d. over all $n$ and all $x$. Define also, for each $n$, the set of 'potentially infected' sites $Y_{n+1}$ at time $n+1$, given by

$$
Y_{n+1}=\bigcup_{\left\{x: n+x \text { even, } X_{n}(x)=1\right\}}\left(x+\eta_{n+1, x}\right) .
$$

Then, for $x \in Y_{n+1}$ such that $X_{n}(x)=0$, we take $X_{n+1}(x)=1$ with probability $q$ and $X_{n+1}(x)=0$ with probability $1-q$, independently of all else; for $x \in Y_{n+1}$ such that $X_{n}(x)=-1$, we take $X_{n+1}(x)=1$ with probability 1 . For $x \notin Y_{n+1}$, we take $X_{n+1}(x)=-1$ if $X_{n}(x)=-1$ and $X_{n+1}(x)=0$ otherwise. (Note that, by induction, these dynamics do indeed imply the property that, for all $n$ and all $x$, we may only have $X_{n}(x)=1$ when $n+x$ is even. Note also that there is no additional generality in allowing the above probability 1 , that a never previously infected site in the set $Y_{n+1}$ becomes infected, to be replaced by any other probability $q^{\prime} \geq q$ : in such a case we may instead simply redefine the distribution of the random sets $\eta_{n+1, x}$ to correspond to replacing each such set by the empty set with probability $1-q^{\prime}$, independently of all else; we then replace $q^{\prime}$ by 1 , and $q$ by $q / q^{\prime}$, to re-express the model as an instance of that already considered.)

We shall say that the process $\left\{X_{n}\right\}_{n \geq 0}$ survives to time $n$ if $X_{n}(x)=1$ for at least one $x$ and that it survives if it survives to all times $n \geq 0$. We assume that the process $\left\{X_{n}\right\}_{n \geq 0}$ is supercritical, i.e. that, for any $X_{0}$ such that $X_{0}(x)=1$ for at least one $x$, there is a strictly positive probability that the process survives. Note that, if $X_{0}^{\prime}$ is obtained from $X_{0}$ by defining $X_{0}^{\prime}(x)=\max \left(0, X_{0}(x)\right)$ for all $x$, and the resulting process $\left\{X_{n}^{\prime}\right\}_{n \geq 0}$ allowed to evolve as above, then, in this coupling, the survival of the process $\left\{X_{n}^{\prime}\right\}_{n \geq 0}$ implies that of the process $\left\{X_{n}\right\}_{n \geq 0}$. The former process may be viewed as an instance of the basic two-state contact process. It follows in particular that the supercriticality of the present three-state process is equivalent to that of the two-state process obtained as above.

Given the process $\left\{X_{n}\right\}_{n \geq 0}$ for each $n$, define $r_{n}=\max \left\{x: X_{n}(x)=1\right\}$ to be the right endpoint of $X_{n}$ (with, as usual, $r_{n}=-\infty$ when $X_{n}(x) \neq 1$ for all $x$ ). Our interest is in the behaviour of the process $\left\{r_{n}\right\}_{n \geq 0}$ for suitably chosen initial states $X_{0}$. (As usual, this, taken 
together with the corresponding behaviour of the left-endpoint process, characterises the growth of the process $\left\{X_{n}\right\}$.)

3.2.2. Formulation as a stochastic recursion and coupling. We now reformulate the process $\left\{X_{n}\right\}_{n \geq 0}$ as a stochastic recursion (1) as in Section 2. The i.i.d. driving sequence $\left\{\xi_{n}\right\}_{n \geq 1}$ is given, for each $n \geq 0$, by the pair $\xi_{n+1}=\left(\xi_{n+1}^{\prime}, I_{n+1}\right)$. Here $\xi_{n+1}^{\prime}=\left\{\xi_{n+1, z}^{\prime}\right\}_{z \leq 0, z \text { even }}$ and each $\xi_{n+1, z}^{\prime} \subseteq\{-1,1\}$ is a random set with the common distribution of the random sets $\eta_{n+1, x}$

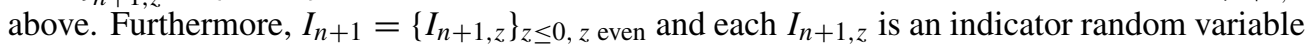
which takes the value 1 with probability $q$ and is 0 otherwise. For each $n$, the random elements $\xi_{n+1}^{\prime}$ and $I_{n+1}$ are independent; furthermore, the random sets $\xi_{n+1, z}^{\prime}$ are independent over all $z$, as are also the random variables $I_{n+1, z}$. The process $\left\{X_{n}\right\}_{n \geq 0}$ is now updated as described above, via the stochastic recursion (1), taking, for each $n$ and $x$,

$$
\eta_{n+1, x}=\xi_{n+1, x-r_{n}}^{\prime},
$$

analogously to (20). Furthermore, given $n$, let $r_{n+1}^{\prime}$ be the rightmost point of the set $Y_{n+1}$ defined above; in the case where $x^{\prime} \in Y_{n+1}$ is such that $X_{n-1}\left(x^{\prime}\right)=0$ or $X_{n-1}\left(x^{\prime}\right)=1$, we take

$$
X_{n+1}\left(x^{\prime}\right)=I_{n+1, x^{\prime}-r_{n+1}^{\prime}},
$$

while, for $x^{\prime} \in Y_{n+1}$ such that $X_{n-1}\left(x^{\prime}\right)=-1$, we already have $X_{n+1}\left(x^{\prime}\right)=1$.

For each $n \geq 0$, let $\left\{\hat{X}_{n^{\prime}}^{(n)}\right\}_{n^{\prime} \geq n}$ be any version of the process $\left\{X_{n^{\prime}}\right\}_{n^{\prime} \geq n}$, started at time $n$ and defined through the above stochastic recursion (1) using (22) and (23) (with $\hat{X}_{n^{\prime}}^{(n)}$ replacing $\left.X_{n^{\prime}}\right)$, in which $\hat{X}_{n}^{(n)}(0)=1$ and $\hat{X}_{n}^{(n)}(x)=-1$ for all $x>0$. Let $\left\{\hat{r}_{n^{\prime}}^{(n)}\right\}_{n^{\prime} \geq n}$ and $\left\{\hat{l}_{n^{\prime}}^{(n)}\right\}_{n^{\prime} \geq n}$ respectively be the left- and right-endpoint processes associated with this process (i.e. $\hat{r}_{n^{\prime}}^{(n)}=$ $\max \left\{x: \hat{X}_{n^{\prime}}^{(n)}(x)=1\right\}$, with $\hat{r}_{n^{\prime}}^{(n)}=-\infty$ if no such $x$ exists, and $\hat{l}_{n^{\prime}}^{(n)}=\min \left\{x: \hat{X}_{n^{\prime}}^{(n)}(x)=1\right\}$, with $\hat{l}_{n^{\prime}}^{(n)}=\infty$ if no such $x$ exists). (Note that, for each $n^{\prime} \geq n$, it is only possible to have $\hat{X}_{n^{\prime}}^{(n)}(x)=1$ at those sites $x$ such that $n^{\prime}-n+x$ is even. Since our various processes will eventually be coupled starting from their right endpoints, this is as it ought to be.)

Define also the particular version $\left\{X_{n^{\prime}}^{(n)}\right\}_{n^{\prime} \geq n}$ of the above process, in which

$$
X_{n}^{(n)}(x)= \begin{cases}1 & \text { if } x=0 \\ 0 & \text { if } x<0 \\ -1 & \text { if } x>0\end{cases}
$$

Also, let $\left\{r_{n^{\prime}}^{(n)}\right\}_{n^{\prime} \geq n}$ and $\left\{l_{n^{\prime}}^{(n)}\right\}_{n^{\prime} \geq n}$ respectively denote the associated right- and left-endpoint processes. Note that it follows from the skip-free property of the dynamics of the process $\left\{X_{n^{\prime}}^{(n)}\right\}_{n^{\prime} \geq n}$ that, for all $n^{\prime}$ to which this process survives,

$$
X_{n^{\prime}}^{(n)}(x) \neq-1 \quad \text { for all } x \leq r_{n^{\prime}}^{(n)}, \quad X_{n^{\prime}}^{(n)}(x)=0 \quad \text { for all } x<l_{n^{\prime}}^{(n)} .
$$

We now require the following lemma, which is a simple generalisation of the classical result for the nearest-neighbour two-state contact process on $\mathbb{Z}$, and which gives the basic coupling on which the application of the theory of Section 2 depends.

Lemma 1. For any instance of the process $\left\{\hat{X}_{n^{\prime}}^{(n)}\right\}_{n^{\prime} \geq n}$ defined above and for the particular instance given by $\left\{X_{n^{\prime}}^{(n)}\right\}_{n^{\prime} \geq n}$ for each $n^{\prime}$ to which the latter process survives,

$$
\hat{X}_{n^{\prime}}^{(n)}(x)=X_{n^{\prime}}^{(n)}(x) \text { for all } x \geq l_{n^{\prime}}^{(n)} .
$$

In particular, we have $\hat{r}_{n^{\prime}}^{(n)}=r_{n^{\prime}}^{(n)}$ for all $n^{\prime}$ to which the process $\left\{X_{n^{\prime}}^{(n)}\right\}_{n^{\prime} \geq n}$ survives. 
Proof. The proof is also a simple generalisation of that which is well known for the classical two-state case, and is given by induction on $n^{\prime} \geq n$. Thus, suppose that (25) holds for some particular $n^{\prime} \geq n$ such that the process $\left\{X_{n^{\prime}}^{(n)}\right\}_{n^{\prime} \geq n}$ survives to (at least) time $n^{\prime}+1$. It then follows from the dynamics of the two processes involved, and using the observations in (24), that, for every $x$ such that $X_{n^{\prime}+1}^{(n)}(x)=1$, we also have $\hat{X}_{n^{\prime}+1}^{(n)}(x)=1$. Furthermore, since the random sets $\eta_{n+1, x}$ are subsets of $\{0,1\}$ (the skip-free property of the present model), any site $x \geq l_{n^{\prime}+1}^{(n)}$ such that $\hat{X}_{n^{\prime}+1}^{(n)}(x)=1$ is necessarily infected (at least) by some site $x^{\prime}$ such that $X_{n^{\prime}}^{(n)}\left(x^{\prime}\right)=1$, so that $X_{n^{\prime}+1}^{(n)}(x)=1$ also (i.e. no additional infection can pass from the left of $l_{n^{\prime}}^{(n)}$ at time $n^{\prime}$ to the right of $l_{n^{\prime}+1}^{(n)}$ at time $\left.n^{\prime}+1\right)$. Since we also have $X_{n^{\prime}+1}^{(n)}(x) \neq-1$ and $\hat{X}_{n^{\prime}+1}^{(n)}(x) \neq-1$ for all $x \leq r_{n^{\prime}+1}^{(n)}$, and $\hat{X}_{n^{\prime}+1}^{(n)}(x)=X_{n^{\prime}+1}^{(n)}(x)=-1$ for all $x>r_{n^{\prime}+1}^{(n)}$, then (25) holds with $n^{\prime}$ replaced by $n^{\prime}+1$.

We shall also require below the particular instance $\left\{\bar{X}_{n}\right\}_{n \geq 0}$ of the process $\left\{\hat{X}_{n}^{(0)}\right\}_{n \geq 0}$ defined above (and started at time 0 ), given by

$$
\bar{X}_{0}(x)= \begin{cases}1 & \text { if } x \leq 0 \\ -1 & \text { if } x>0\end{cases}
$$

This process is useful since, a.s., it survives for all time. This enables us to make some necessary definitions without an a priori need to condition on survival. Also, define $\left\{\bar{r}_{n}\right\}_{n \geq 0}$ to be the right-endpoint process associated with the process $\left\{\bar{X}_{n}\right\}_{n \geq 0}$.

3.2.3. Definition of events $F_{n}$ and times $\tau_{k}$. We define the sequence of events $\left\{F_{n}\right\}_{n \in \mathbb{Z}_{+}}$of Section 2, analogously to Section 3.1 , by saying that the event $F_{n}$ occurs if and only if the process $\left\{X_{n^{\prime}}^{(n)}\right\}_{n^{\prime} \geq n}$ survives for all future time, i.e. $r_{n^{\prime}}^{(n)}>-\infty$ for all $n^{\prime} \geq n$. It again follows from the definition of the process $\left\{X_{n^{\prime}}^{(n)}\right\}_{n^{\prime} \geq n}$ that the sequence $\left\{F_{n}\right\}_{n \in \mathbb{Z}_{+}}$satisfies conditions (F1) and (F2) of Section 2. (That the common value $p$, say, of $\mathbb{P}\left(F_{n}\right)$ is strictly positive follows once more from our earlier assumption of supercriticality.)

For each $n \geq 0$, define the event

$$
H_{n}=\left\{\bar{X}_{n}(x)=-1 \text { for all } x>\bar{r}_{n}\right\}
$$

(where $\left\{\bar{X}_{n}\right\}_{n \geq 0}$ is the process defined above with initial state $\bar{X}_{0}$ given by (26)). Note that an equivalent definition is that $H_{n}=\left\{\bar{r}_{n} \geq \bar{r}_{n^{\prime}}\right.$ for all $\left.n^{\prime}<n\right\}$, i.e. that $n$ is such that at time the right-endpoint process $\left\{\bar{r}_{n}\right\}_{n \geq 0}$ is at a record value. Note also that the event $H_{0}$ always occurs, and further that, for all $n$, we have $H_{n} \in \sigma_{n}$.

We define the sequence of events $\left\{A_{n}\right\}_{n \geq 0}$ of Theorem 1 by, for each $n, A_{n}=H_{n} \cap F_{n}$. As usual the random times $\tau_{k}$ are the successive occurrence times of the events $A_{n}$. (The more complex definition of the events $A_{n}$, in comparison with that of Section 3.1, is required to make the right-endpoint couplings below work correctly.)

We can now state and prove the following analogue of Proposition 1.

Theorem 3. The successive (segments of) processes $\left\{\xi_{\tau_{k}+1}, \ldots, \xi_{\tau_{k+1}}\right\}, k \geq 0$, are i.i.d. Furthermore, the distribution of each of these lengths is light tailed, i.e. geometrically bounded, and in particular possesses moments of all orders.

Proof. We show first that the sequences of events $\left\{F_{n}\right\}_{n \geq 0}$ and $\left\{H_{n}\right\}_{n \geq 0}$ defined above are such that the conditions of Corollary 1 are satisfied. Note first that it follows from Lemma 1 
(applied at the time $n$ ) that, for any $n$ such that the event $H_{n}$ occurs,

$$
\bar{r}_{n^{\prime}}=\bar{r}_{n}+r_{n^{\prime}}^{(n)} \text { for all } n^{\prime} \geq n \text { to which the process }\left\{X_{n^{\prime}}^{(n)}\right\}_{n^{\prime} \geq n} \text { survives. }
$$

We first show condition (b) of Corollary 2. Fix $n \geq 0$ and $m>0$. Given that the event $A_{n}$ occurs (which implies both the occurrence of the event $H_{n}$ and the survival for all time of the process $\left\{X_{n^{\prime}}^{(n)}\right\}_{n^{\prime} \geq n}$, it follows from (27) that the event $H_{n+m}$ occurs if and only if there occurs the event $E_{n, n+m}^{\prime \prime \prime}$ that the process $\left\{r_{n^{\prime}}^{(n)}\right\}_{n^{\prime} \geq n}$ is at a record value at time $n+m$, i.e. $r_{n+m}^{(n)} \geq r_{n+m^{\prime}}^{(n)}$ for all $0 \leq m^{\prime}<m$. Condition (b) of Corollary 2 is now immediate, since the array $\left\{E_{n, n+m}^{\prime \prime}\right\}_{n \geq 0, m>0}$ trivially possesses the properties required by that condition. For condition (a) of Corollary 2 , again fix $n \geq 0$ and $m>0$. Given that the event $H_{n} \cap A_{n+m}$ occurs, it follows from (27) (both as stated and with $n$ replaced by $n+m)$ that the event $A_{n}$ occurs if and only if there occurs the event $E_{n, n+m}^{\prime}$ that the process $\left\{X_{n^{\prime}}^{(n)}\right\}_{n^{\prime} \geq n}$ survives to time $n+m$. Condition (a) of Corollary 2 now follows, since again the array $\left\{E_{n, n+m}^{\prime}\right\}_{n \geq 0, m>0}$ trivially possesses the properties required by that condition. Thus, the first statement of the present theorem follows from Corollary 2 .

In order to prove the second statement, define the random time $\tau^{\prime}=\left\{\min n \geq 1: A_{n}\right.$ occurs $\}$. As in the corresponding argument for the second statement of Proposition 1, it is sufficient to show that the (unconditional) distribution of $\tau^{\prime}$ is geometrically bounded.

We first show that if $\rho$ is the first time to which the process $\left\{X_{n}^{(0)}\right\}_{n \geq 0}$ fails to survive then, as for the basic two-state contact process, there exists $\alpha>0$ such that

$$
\mathbb{P}(n \leq \rho<\infty) \leq \mathrm{e}^{-\alpha n}, \quad n \geq 0
$$

For each $n \geq 0$, define the process $\left\{\tilde{X}_{n^{\prime}}^{(n)}\right\}_{n^{\prime} \geq n}$ via the above stochastic recursion (1) and (22), (23), with the initial state $\tilde{X}_{n}^{(n)}$ given by $\tilde{X}_{n}^{(n)}(0)=1$ and $\tilde{X}_{n}^{(n)}(x)=0$ for all other $x$. Let $\tilde{\rho}$ be the minimum value of $n \geq 0$ such that the process $\left\{\tilde{X}_{n^{\prime}}^{(n}\right\}_{n^{\prime} \geq n}$ (started at time $n$ ) survives. Suppose now that the process $\left\{X_{n^{\prime}}^{(0)}\right\}_{n^{\prime} \geq 0}$ survives to time $\tilde{\rho}$; then, in yet another instance of the coupling arguments used above (in which processes are 'matched' from their right endpoints), it follows from the above stochastic recursion that the process $\left\{X_{n^{\prime}}^{(0)}\right\}_{n^{\prime} \geq 0}$ necessarily survives for all time. We deduce that if $\rho<\infty$ then necessarily $\rho<\tilde{\rho}$, and, thus, we conclude that, for all $n \geq 0$,

$$
\mathbb{P}(n \leq \rho<\infty) \leq \mathbb{P}(\tilde{\rho}>n) .
$$

However, given the driving sequence $\left\{\xi_{n}\right\}_{n \geq 1}$, the successive processes $\left\{\tilde{X}_{n^{\prime}}^{(n)}\right\}_{n^{\prime} \geq n}$ are simply successive instances of the basic two-state nearest-neighbour contact process, in each case started with a single infective, launched by the sequence $\left\{\xi_{n}\right\}_{n \geq 1}$ exactly as in Section 3.1. We have already observed in that section that the time required to initiate such a process which survives (the time to the first of the events $F_{n}$ of that section) is geometrically bounded. The required conclusion (28) now follows from this and (29).

To complete the proof, we need to show that the distribution of $\tau^{\prime}$ is geometrically bounded. We argue as in Section 3.1, again with a little extra complication. We again proceed forward in time, starting at time $v_{1}=1$ and checking at that, and at selected subsequent times $v_{k}, k>1$, such that the event $H_{v_{k}}$ occurs, whether the event $F_{v_{k}}$ also occurs; if it fails to do so, we wait until the process $\left\{X_{n^{\prime}}^{\left(v_{k}\right)}\right\}_{n^{\prime} \geq v_{k}}$ dies out—which we have shown it then does in a time which is geometrically bounded-before resuming checking for $F_{v_{k+1}}$ at the first subsequent time $v_{k+1}$ such that $H_{v_{k+1}}$ occurs. It follows, again from the right-endpoint coupling of Lemma 1 as in the first part of the present proof, that, for each such $k \geq 1$, the increment $\bar{r}_{v_{k+1}}-\bar{r}_{v_{k}}$ is equal to the maximum value attained by the right endpoint of the process $\left\{X_{n^{\prime}}^{\left(v_{k}\right)}\right\}_{n^{\prime} \geq v_{k}}$ prior to its dying out, and so this increment is geometrically bounded. Furthermore, from the construction, the successive increments $\bar{r}_{v_{k+1}}-\bar{r}_{v_{k}}$ are i.i.d. Let $K$ be the number of checks required to obtain a 
success (the event $F_{v_{K}}$ occurs). Then, since each of the above checks is independently successful with probability $p>0$, the random variable $K$ is geometrically distributed, independently of the above increments in the right-endpoint process $\left\{\bar{r}_{n}\right\}$. Thus, $\bar{r}_{v_{K}}-\bar{r}_{1}$ is a geometric sum of i.i.d. geometrically bounded random variables, and so $\bar{r}_{v_{K}}$ is geometrically bounded.

Now let $\left\{\tilde{r}_{n}\right\}_{n \geq 0}$ be the right endpoint of the three-state process $\left\{\tilde{X}_{n}\right\}_{n \geq 0}$ in which $\tilde{X}_{0}(0)=1$ and $\tilde{X}_{0}(x)=0$ for $x \neq 0$. Then, from the usual coupling $\tilde{r}_{n} \leq \bar{r}_{n}$ for all $n$, and so $\tilde{r}_{v_{K}}$ is also geometrically bounded. However, $\left\{\tilde{X}_{n}\right\}_{n \geq 0}$ is simply an instance of the supercritical two-state contact process, in which the set of initially infected sites is $\mathbb{Z}_{-}$. It follows easily from the results of Kuczek for the regenerative behaviour of this process (as given in the previous section) that, since $\tilde{r}_{v_{K}}$ is geometrically bounded, the random variable $v_{K}$ is itself geometrically bounded. Since $v_{K}$ is the time $n$ to the occurrence of some event $A_{n}$, the result that $\tau^{\prime}$ is geometrically bounded now follows.

Finally, consider again any instance $\left\{\hat{X}_{n}\right\}_{n \geq 0}$ of our three-state process, defined by the stochastic recursion (1) using (22) and (23) as above, in which the initial state $\hat{X}_{0}$ is such that $\hat{X}_{0}\left(x_{0}\right)=1$ for some $x_{0}$ and $\hat{X}_{0}(x)=-1$ for all $x>x_{0}$. Again, let $\hat{r}_{n^{\prime}}=\max \left\{x: \hat{X}_{n^{\prime}}(x)=1\right\}$ be its associated right-endpoint process. Let $F$ be the event that the process $\left\{\hat{X}_{n}\right\}_{n \geq 0}$ survives. It follows from the earlier coupling for this process (with the time $\tau_{0}$ replacing the time 0 ) that event $F$ occurs if and only if the process $\left\{\hat{X}_{n}\right\}_{n \geq 0}$ survives to time $\tau_{0}$. Analogously to the situation for the two-state process, we have $F_{0} \subseteq F$, and, conditional on the event $F$ and for all $k \geq 0$,

$$
\hat{r}_{\tau_{k}+n^{\prime}}=\hat{r}_{\tau_{0}}+\sum_{j=0}^{k-1} r_{\tau_{j+1}}^{\left(\tau_{j}\right)}+r_{\tau_{k}+n^{\prime}}^{\left(\tau_{k}\right)} \quad \text { for all } n^{\prime} \geq 0 .
$$

Thus, again as for the two-state process, we have the following corollary to Theorem 3.

Corollary 5. For the process $\left\{\hat{X}_{n}\right\}_{n \geq 0}$ and on the set $F$ defined above, the successive (segments of) processes $\left\{\hat{r}_{\tau_{k}+1}, \ldots, \hat{r}_{\tau_{k+1}}\right\}, k \geq 0$, are i.i.d. Furthermore, for some constant $\mu$,

$$
\frac{\hat{r}_{n}}{n} \rightarrow \mu \text { a.s. as } n \rightarrow \infty
$$

and, in the Skorokhod topology,

$$
\frac{\hat{r}_{[n t]}-n t \mu}{\sqrt{n}} \rightarrow B(t) \text { in distribution as } n \rightarrow \infty,
$$

where again B is Brownian motion with some nontrivial diffusion constant.

Similar behaviour holds for the left-endpoint process for any process $\left\{X_{n}\right\}_{n \geq 0}$ whose initial state $X_{0}$ is such that $X_{0}\left(x_{0}\right)=1$ for some $x_{0}$ and $X_{0}(x)=-1$ for all $x<x_{0}$. Thus, finally, for any process $\left\{X_{n}\right\}_{n \geq 0}$ whose initial state $X_{0}$ is such that $X_{0}(x)=-1$ for all $x$ outside some finite interval, we may deduce the behaviour, conditional on its survival, of both its left and right endpoints. In particular, conditional on its survival, the growth rate of the process is given by $2 \mu$, where $\mu$ is as given by Corollary 5 .

\section{Infinite-bin models}

In this section we consider a discrete-space infinite-bin model and its continuous-space analogue.

In the discrete setting, we review the basic model introduced and studied in [13] (see also [10] and [15]). We recall a stability result from [13] (see Proposition 2 below), with a new 
proof, and provide a new generalisation (see Theorem 4). We show that the both results may be considered as applications of the techniques developed in Section 2. Then we introduce a new continuous-space model and prove a new stability result there (see Theorem 5), by applying again the methodology from Section 2.

\subsection{Discrete-space infinite-bin model}

4.1.1. Basic model. Consider an infinite number of bins arranged on the line and indexed, say, by the nonpositive integers. Each bin can contain an unlimited number of particles (we assume it to be finite for the moment). A configuration is either a finite-dimensional vector $\boldsymbol{x}=\left(x_{-l}, \ldots, x_{0}\right)$, where $x_{i}$ is the number of particles in bin $i$, or an infinite-dimensional vector $\boldsymbol{x}=\left(\ldots, x_{-l}, \ldots, x_{0}\right)$.

The indexing by nonpositive integers is convenient because we are interested in the asymptotic behaviour of a finite number of rightmost coordinates of vectors representing a stochastic recursion. At each integer step, precisely one particle - the active particle-of the current configuration is chosen according to some rule (to be given below). If the particle is in bin $-i \leq-1$ then a new particle is created and placed in bin $-i+1$. Otherwise, if the chosen particle is in bin 0 then a new bin is created to hold the 'child particle' and a relabelling of the bins occurs: the existing bins are shifted by one place to the left (are re-indexed) and the new bin is given the label 0 .

To be more precise, define the configuration space $\mathcal{X}$ as the set of all infinite-dimensional vectors $\boldsymbol{x}=\left(\ldots, x_{-2}, x_{-1}, x_{0}\right)$ with nonnegative integer-valued coordinates, which have the following property: if $x_{-l}>0$ then $x_{-l+1}>0$. In other words, either all the coordinates of a configuration vector are strictly positive or there is only a finite number of nonzero coordinates, say $l+1$-then these are coordinates $x_{-l}, x_{-l+1}, \ldots, x_{0}$. We endow $\mathcal{X}$ with the natural topology of pointwise convergence, and let $\mathscr{B}_{x}$ be the corresponding class of Borel sets generated by this topology.

The extent of an $\boldsymbol{x} \in \mathcal{X}$ is defined as $|\boldsymbol{x}|=l$ if there is $l+1$ nonzero coordinates, $\boldsymbol{x}=$ $\left(\ldots, 0,0, x_{-l}, \ldots, x_{0}\right)$, with the $\mathcal{L}_{1}$-norm

$$
\|x\|=\sum_{j=0}^{l} x_{-j},
$$

and if all the coordinates of $\boldsymbol{x}$ are positive, we set $|\boldsymbol{x}|=\|\boldsymbol{x}\|=+\infty$.

Let $\mathbb{N}$ be the set of positive integers. The dynamics of the model may be defined using the map $f: \mathcal{X} \times \mathbb{N} \rightarrow \mathcal{X}$, where

$$
f(\boldsymbol{x}, \xi)= \begin{cases}{[\boldsymbol{x}, 1]} & \text { if } \xi \leq x_{0}, \\ \boldsymbol{x}+\boldsymbol{e}_{-k} & \text { if } \sum_{j=0}^{k} x_{-j}<\xi \leq \sum_{j=0}^{k+1} x_{-j}, 0 \leq k<|\boldsymbol{x}|, \\ \boldsymbol{x}+\boldsymbol{e}_{-|\boldsymbol{x}|} & \text { if } \xi>\|\boldsymbol{x}\| .\end{cases}
$$

Here $[\boldsymbol{x}, 1]$ is a concatenation of the vector $\boldsymbol{x}$ with 1 , i.e. if $\boldsymbol{x}=\left(\ldots, x_{-l}, \ldots, x_{0}\right)$ then $[\boldsymbol{x}, 1]=$ $\left(\ldots, y_{-l-1}, y_{-l}, \ldots, y_{-1}, y_{0}\right)$, where $y_{0}=1$ and $y_{-j-1}=x_{-j}$ for $j \geq 0$. Furthermore, $\boldsymbol{e}_{-j}$ is the infinite unit vector whose $-j$ th coordinate is 1 with all other coordinates equal to 0 . Then, given an i.i.d. sequence $\left\{\xi_{n}\right\}_{n \in \mathbb{Z}_{+}}$of $\mathbb{N}$-valued random variables and an $\mathcal{X}$-valued random variable $X_{0}=\left(\ldots, X_{-k, 0}, X_{-k+1,0}, \ldots, X_{-1,0}, X_{0,0}\right)$, we define a stochastic recursion by

$$
X_{n+1}=f\left(X_{n}, \xi_{n+1}\right), \quad n \geq 0,
$$

where $X_{n}=\left(\ldots, X_{-k, n}, X_{-k+1, n}, \ldots, X_{-1, n}, X_{0, n}\right)$. 
In words, the dynamics may be explained as follows. Each time $n$, we number again the existing particles from the rightmost bin to the leftmost (so, if $X_{n}$ takes value $\boldsymbol{x}=$ $\left(\ldots, 0, x_{-l}, \ldots, x_{0}\right)$ then the particles in the rightmost bin are numbered 1 to $x_{0}$, in the next bin they are numbered $x_{0}+1$ to $x_{0}+x_{-1}$, and so on). Then the random variable $\xi_{n}$ is the number of the active particle defined in the earlier description.

Fix a nonnegative integer $k$, and let $X_{n}(-k)$ be the $(k+1)$-dimensional projection of $X_{n}$,

$$
X_{n}(-k)=\left(X_{-k, n}, X_{-k+1, n}, \ldots, X_{0, n}\right) .
$$

The following result may be found in [13].

Proposition 2. Assume that $\left\{\xi_{n}\right\}_{n \in \mathbb{Z}_{+}}$is an i.i.d. sequence. Assume also that $\mathbb{P}\left(\xi_{i}=1\right)>0$ and $\mathbb{E} \xi_{i}<\infty$. Then, for any integer $k \geq 0, X_{n}(-k)$ converges to a proper limiting random vector in the total variation norm. Therefore, $X_{n}$ weakly converges to its proper limit.

Based on the theory from Section 2, we can provide a short alternative proof of Proposition 2. We start with the simplest case $k=0$. In this case, the proof is based on Corollaries 1 and 3 .

In order to avoid trivialities, assume that $\mathbb{P}\left(\xi_{i}=1\right)<1$.

Let the functions $R_{i}$ of Section 2 be given by $R_{i}\left(X_{n+i}, X_{n}\right)=X_{0, n+i}$. Furthermore, let

$$
F_{n}=\bigcap_{i \geq 1}\left\{\xi_{n+i} \leq i\right\} \equiv \bigcap_{i \geq 1} F_{n, i} .
$$

Note that, since $\mathbb{E} \xi_{i}<\infty$, the events $F_{n}$ have strictly positive probability, and indeed satisfy conditions (F1) and (F2) of Section 2. Define now, for each $n$, the event $A_{n}=F_{n}$. Clearly,

$$
F_{n} \cap F_{n+m}=\bigcap_{i=1}^{m} F_{n, i} \cap F_{n+m},
$$

and so condition (7) of Corollary 1 holds. Furthermore, condition (15) of Theorem 2 holds because, given the event $F_{n}$, the future process of placing particles is the same for all histories up to time $n$.

Finally, the aperiodicity condition of Corollary 3 follows since

$$
\begin{aligned}
\mathbb{P}\left(\tau_{n+1}-\tau_{n}=1\right) & =\sum_{l} \mathbb{P}\left(\tau_{n}=l, \tau_{l+1}=l+1\right) \\
& =\sum_{l} \mathbb{P}\left(\tau_{n}=l\right) \mathbb{P}\left(F_{l+1} \mid F_{l}\right) \\
& =\mathbb{P}\left(F_{1} \mid F_{0}\right) \\
& =\prod_{i=2}^{\infty} \mathbb{P}\left(\xi_{i} \leq i-1 \mid \xi_{i} \leq i\right) \\
& \geq \prod_{i=2}^{\infty} \mathbb{P}\left(\xi_{i} \leq i-1\right) \\
& >0
\end{aligned}
$$

The proof of Proposition 2 now follows in the case $k=0$ from Corollaries 1 and 3 . 
For the proof of general $k>0$, we need events of the form $B_{n}=H_{n} \cap F_{n}$, where the events $F_{n}$ are again as given by (30) and

$$
H_{n}=\bigcap_{1 \leq i \leq k}\left\{\xi_{n+1-i}=1\right\}
$$

We may observe that, given $H_{n}$, we have $X_{n}(-k)=(1,1, \ldots, 1)$.

Now we define the events $A_{n}$ as follows: $A_{n}=\varnothing$ for $n<2 k$ and, for $n \geq 2 k$,

$$
A_{n}=\bigcap_{i=1}^{k-1} B_{n-i}^{\mathrm{c}} \cap B_{n},
$$

which may be represented as $A_{n}=E_{n-k, n} \cap F_{n}$ for a stationary sequence $E_{n-k, n} \in \sigma_{n-k+1, n}$. Finally, we may take $R_{i}\left(X_{n+i}, X_{n}\right)=X_{n+i}(-k)$. Then all conditions of Theorem 2 are satisfied, and the result again follows from Corollary 3 , on noting that once more aperiodicity follows from the condition $\mathbb{P}\left(\xi_{i}=1\right)>0$.

Remark 5. This model has close links to the model from [9]; see [13] for more details.

4.1.2. Extension of the basic model. Consider the infinite-bin model introduced in Section 4.1.1, and let $p_{i}=\mathbb{P}(\xi=i)$. One of the main conditions in Proposition 2 is that $p_{1}>0$. We assume now that this condition is violated and that instead the following condition holds: there exist two positive integers $1<i_{1}<i_{2}$ such that

$$
p_{i_{1}}>0 \text { and } p_{i_{2}}>0 .
$$

Then the following statement holds.

Theorem 4. Assume that $\left\{\xi_{n}\right\}_{n \in \mathbb{Z}_{+}}$is an i.i.d. sequence with a common finite mean $\mathbb{E} \xi_{i}$. Assume also that $p_{1}=0$ and that condition (31) holds. Assume further that the numbers $i_{1}$ and $i_{2}$ are mutually prime. Then, for any integer $k \geq 0, X_{n}(-k)$ converges to a proper limiting random vector in the total variation norm. Therefore, $X_{n}$ weakly converges to its proper limit.

The proof of Theorem 4 will be based on the following simple observation (see, e.g. [8]).

Lemma 2. For any two integers $1<i_{1}<i_{2}$, there exist a positive integer $m$ and a sequence of integers $j_{1}, j_{2}, \ldots, j_{m-1} \in\left\{i_{1}, i_{2}\right\}$ such that, for any $n>m$ and any vector $X_{n-m}$ as in Section 4.1.1, we have

$$
X_{n, 0} \mathbf{1}_{B_{n}} \geq i_{1} \mathbf{1}_{B_{n}} \quad \text { a.s. }
$$

where the events $B_{n}$ are defined as

$$
B_{n}=\left\{\xi_{n}=i_{2}\right\} \cap \bigcap_{l=1}^{m-1}\left\{\xi_{n-m+l}=j_{l}\right\} \cap\left\{\xi_{n-m}=i_{2}\right\} .
$$

Proof of Theorem 4. By the conditions of the theorem, the stationary events $B_{n}$ defined by (32) have a positive probability.

Let $r=j_{1}(k+1)$. For $n \leq r$, we let $A_{n}=\varnothing$. For $n>r$, let $A_{n}=H_{n} \cap F_{n}$, with

$$
H_{n}=B_{n-r} \cap D_{n}, \quad \text { where } \quad D_{n}=\bigcap_{1 \leq l \leq r}\left\{\xi_{n+1-l}=i_{1}\right\},
$$

and where

$$
F_{n}=\bigcap_{l \geq 1}\left\{\xi_{n+l} \leq i_{1}+l-1\right\} .
$$


Clearly, for $n>r$, given the occurrence of the event $H_{n}$, all the coordinates of the vector $X_{n}(-k)$ are equal to $i_{1}$. Thus, given the event $A_{n}$, the placings of the particles numbered $n+1, n+2, \ldots$ do not depend on the left tail of the vector $X_{n}$ or on the past values of the vector $X_{j}, j<n$.

One can check directly that both conditions (9) and (10) are satisfied.

We may now define the functions $R_{i}$ of Section 2 by $R_{i}\left(X_{n+i}, X_{n}\right)=X_{n+i}(-k)$. Then condition (15) holds, which implies conclusion (16) of Theorem 2(a).

Observe further that $B_{n+l} \cap D_{n}=\varnothing$ for any $1 \leq l \leq r$. Therefore, $A_{n} \cap A_{n^{\prime}}=\varnothing$ for all $n<n^{\prime}$ with $n^{\prime}-n \leq r+i_{2}-i_{1}$ and $\mathbb{P}\left(A_{n} \cap A_{n^{\prime}}\right)>0$ if $n^{\prime}-n>r+i_{2}-i_{1}$. The latter implies the aperiodicity condition of Corollary 3 , and the required result follows.

\subsection{Continuous-space model with varying link lengths}

In this section we introduce and study a new model which is a continuous-space extension of the infinite-bin model, and which has applications in, for example, queueing theory. As described below, it may be viewed as a model for the locations of points on the negative real line, in which at each successive time precisely one of these points gives birth to a further point, and in which it is convenient to associate a link between this child point and its parent. We thus think of it as a random links model. Once again, our aim is to study the asymptotic behaviour of this model as 'seen from the right'.

Before introducing the new model, we remark that the basic model of Section 4.1.1 may be described slightly differently. Namely, we may assume that, at each time $n$, particle number $-j$ may be active with some probability, say $p(-j)$. Each active particle proposes to put a new particle in the bin next to its own (in other words, at distance 1 to the right), and the rightmost active particle wins. If particles become active independently then this description coincides with the description proposed earlier if we let $\mathbb{P}(\xi>j)=\prod_{i=1}^{j}(1-p(-i))$.

Now assume, for simplicity, that all the $p(-j)$ are equal and introduce the following continuous-space extension of the model, in which the positions of particles are real valued: at time $n$, each active particle (say particle $-j$ ) proposes a location for the new particle at a random distance, $l_{n,-j}$ to the right of particle $-j$ (here the $l_{n,-j}$ needs not be integer), and the rightmost proposed location (say that proposed by particle $-j_{0}$ ) wins. Then we say there is a link of length $l_{n,-j_{0}}$ from particle $-j_{0}$ to the new particle.

Remark 6. One may view this model as a model of a system with infinitely many servers and with random constraints. There is an infinite queue in front; each successive client $n$ is allocated to a free server, but the start of its service is delayed by the maximum of times $l_{n,-j}$ of all previous clients that are active; see e.g. [13] and the references therein for further comments.

Remark 7. One can consider various natural generalisations of this setting where the same methodology may be easily applied. For example, we may assume that, at any time, the first $K \geq 0$ particles cannot be active and that all the others become active independently, with either equal probabilities $p \in(0,1]$ or varying probabilities.

Here is a formal description of the model. Let $\mathcal{X}$ be the space of left-infinite vectors of the form $\boldsymbol{x}=\left(\ldots, x_{-k}, x_{-k+1}, \ldots, x_{-1}, x_{0}\right)$, where $x_{0}=0$ and $x_{-k} \leq x_{-k+1}$ for all $k \geq 1$. We also assume that either all the coordinates of $x$ are finite or some of them are equal to $-\infty$. In the latter case, due to the monotonicity, there will be only a finite number of finite coordinates, say, $x_{-j}=-\infty$ for all $j>k$ and $x_{-j}>-\infty$ for all $j \leq k$ for some $k=0,1, \ldots$. Then we write $\boldsymbol{x}=\left(x_{-k}, \ldots, x_{0}\right)$ for short. We denote by $\chi_{0}$ the space of finite-dimensional vectors (which may be viewed as a subspace of $\mathcal{X}$ ). 
Let $\mathcal{L}$ be the space of infinite sequences $\boldsymbol{l}=\left(\ldots, l_{-k} . l_{-k+1}, \ldots, l_{0}\right)$ consisting of nonnegative real-valued elements, and let $\mathcal{Q}$ be the space of infinite sequences of the form $\boldsymbol{q}=$ $\left(\ldots, q_{-k}, q_{-k+1}, \ldots, q_{0}\right)$, where each $q_{-k} \in\{0,1\}$.

Introduce the function

$$
f: X_{0} \times \mathcal{L} \times \mathcal{Q} \rightarrow \mathcal{X}_{0}
$$

using the following rule. For $\boldsymbol{x}=\left(x_{-k}, \ldots, x_{0}\right)$, let

$$
h:=h(\boldsymbol{x}, \boldsymbol{l}, \boldsymbol{q})=\max _{\left\{i: q_{-i}=1\right\}}\left(x_{-i}+l_{-i}\right)
$$

and

$$
h:=h(\boldsymbol{x}, \boldsymbol{l}, \boldsymbol{q})=x_{-k}
$$

if $q_{-i}=0$ for all $0 \leq i \leq k$.

If $h \leq 0$ and, say, $x_{-j} \leq h \leq x_{-j+1}$ for some $j$, then

$$
f(\boldsymbol{x}, \boldsymbol{l}, \boldsymbol{q})=\left(x_{-k}, \ldots, x_{-j}, h, x_{-j+1}, \ldots, x_{0}\right)
$$

and if $h>0$ then

$$
h(\boldsymbol{x}, \boldsymbol{l}, \boldsymbol{q})=\left(x_{-k}-h, x_{-k+1}-h, \ldots, x_{0}-h, 0\right) .
$$

In other words, if $h \leq 0$, we add an extra coordinate $h$, and if $h>0$, we again add the coordinate and then subtract $h$ from all coordinates of the new vector.

Remark 8. An equivalent way to describe the dynamics is to use point processes. Instead of considering vectors, we may consider finite-ordered sequences of points, with the rightmost point at 0 .

Now we introduce stochastic assumptions. Let $\left\{\boldsymbol{l}_{n}\right\}$ and $\left\{\boldsymbol{q}_{n}\right\}$ be two i.i.d. sequences of vectors that do not depend on each other. Assume also that each $\mathbf{l}_{n}$ and each $\boldsymbol{q}_{n}$ consists of i.i.d. random variables, $l_{n, j}$ and $q_{n, j}$. Let $q=\mathbb{P}\left(q_{n, j}=0\right)=1-p$ with $p=\mathbb{P}\left(q_{n, j}=1\right)$. Assume that $\mathbb{P}\left(l_{0,0}>0\right)=1$ and that

$$
\mathbb{E}\left(l_{0,0}\right)^{2}<\infty
$$

and let

$$
a=\mathbb{E} l_{0,0} .
$$

Recall that vectors $X_{n}$ always have infinitely many coordinates. Our model is now defined by starting from a fixed vector $X_{0} \in \mathcal{X}_{0}$ and running the stochastic recursion

$$
X_{n+1}=f\left(X_{n}, \mathbf{l}_{n}, \boldsymbol{q}_{n}\right) .
$$

Our aim is now to establish the following analogue of Theorem 4 for the discrete-space model.

Theorem 5. For any $j \geq 0$, the finite-dimensional projections $\left(X_{n,-j}, \ldots, X_{n, 0}\right)$ of vectors $X_{n}$ converge to a proper limiting vector in the total variation norm.

Proof. Let $v_{n}=\min \left\{i: q_{n,-i}=1\right\}$. Then $\left\{v_{n}\right\}$ is an i.i.d. sequence with a common geometric distribution. It is convenient to us to assume this sequence to be doubly infinite, $-\infty<n<\infty$. 
Analogously to Section 4.1.1, introduce the events

$$
F_{n}^{(1)}=\bigcap_{j \geq 1}\left\{v_{n+j} \leq j\right\}
$$

and conclude that these events form a stationary ergodic sequence, each with a strictly positive probability

$$
\mathbb{P}\left(F_{0}^{(1)}\right)=\prod_{j \geq 1}\left(1-q^{j}\right)>0,
$$

and, moreover, satisfy the monotonicity condition (7). Thus, by Corollary 1 , the times $0<$ $T_{1}<T_{2}<\cdots$ of the occurrences of the events $F_{n}^{(1)}$ form a stationary renewal sequence. We may easily extend this sequence to the stationary renewal sequence $\cdots<T_{-1}<T_{0} \leq 0<$ $T_{1}<T_{2}<\cdots$ on the whole real line.

Furthermore, the i.i.d. cycle lengths $t_{k}=T_{k+1}-T_{k}, k \neq 0$, have a light-tailed distribution (i.e. have a finite exponential moment) and, therefore, a finite positive mean $b=\mathbb{E} t_{1}$. Also, the cycle $T_{1}-T_{0}$ has a light-tailed distribution.

Assume for simplicity that the initial vector $X_{0}$ corresponds to a single particle at the origin with all the others at $-\infty$. Number this particle 0 . Each subsequent configuration $X_{n}$ adds precisely one further, finitely located, particle to the configuration $X_{n-1}$ (with the existing particles relocated if necessary). Number this particle $n$. Thus, particles are numbered in the order of their creation, and are assumed to keep their numbering for all subsequent times (including when they are relocated). Now colour 'red' all particles numbered $T_{1}, T_{2}, \ldots$, i.e. those created at the occurrence times of the events $F_{n}^{(1)}$; colour 'green' the remaining particles. For each $n$ and each $k$ such that $n>T_{k+1}$, consider the relative locations of the particles in vector $X_{n}$. The following observations are clear:

- the red particle $T_{k}$ is located to the left of the red particle $T_{k+1}$, and the distance between them is a random variable, say, $d_{k}$ which is stochastically bigger than the 'typical' link $l_{0,0} ;$ in particular, $\mathbb{E} d_{k} \geq a>0 ;$

- all the green particles numbered $T_{k}+1, \ldots, T_{k+1}-1$ are located between these two red particles;

- the relative locations and, in particular, the distances between particles numbered $T_{k}, \ldots$, $T_{k+1}$ stay the same for all $n>T_{k+1}$.

Therefore, if $n=T_{k+1}$ for some $k \geq 0$ then the $t_{k}$ last coordinates of vector $X_{n}$ take values between $-d_{k}$ and 0 , the next (to the left) $t_{k-1}$ coordinates take values between $-d_{k-1}-d_{k}$ and $-d_{k}, \ldots, t_{k}$ coordinates take values between $-d_{1}-d_{2}-\cdots-d_{k}$ and $-d_{2}-\cdots-d_{k}$, and then $T_{1}$ coordinates are smaller than $-d_{k}-\cdots-d_{1}$ (recall that there are also infinitely many coordinates equal to $-\infty$ ). Therefore, the vector $X_{n}$ is smaller (coordinatewise) than the vector, say $Y_{n}$, with infinitely many finite coordinates where the last $t_{k}$ coordinates equal 0 , the next $t_{k-1}$ coordinates equal $-d_{k}, \ldots, t_{1}$ coordinates equal $-d_{2}-\cdots-d_{k}, t_{0}$ coordinates equal $-d_{k}-\cdots-d_{1}, t_{-1}$ coordinates equal $-d_{k}-\cdots-d_{0}$, etc.

Furthermore, we may define vectors $Y_{n}$ for all $n$ (and not only for those with $\mathbf{1}_{F_{n}}(1)=1$ ) as follows: if $T_{k} \leq n<T_{k+1}$ for some $k$, we obtain the vector $Y_{n}$ by concatenating the vector $Y_{T_{k}}$ with $n-T_{k}$ coordinates equal to 0 , i.e. $Y_{n}=\left(\ldots, Y_{T_{k}}, 0,0, \ldots, 0\right)$. Then, clearly, $Y_{n} \geq X_{n}$, coordinatewise, for all $n \geq 0$.

Since the sequence $\left\{T_{n}\right\}$ is stationary and renewal, the vectors $\left\{Y_{n}\right\}$ form a stationary ergodic sequence. For any $n$, let $T_{n, 0} \leq n$ be the last occurrence time of the events $F_{k}^{(1)}$ before or at 
time $n$, let $T_{n,-1}<T_{n, 0}$ the previous such time, and so on. Furthermore, let $d_{n, i}$ be the distance between particles $T_{n, i-1}$ and $T_{n, i}$ in the vector $Y_{n}$. By stationarity, the random variable $T_{n, 0}-n$ has the same distribution as $T_{0}$ and the random vectors $\left\{\left(T_{n, i}-T_{n, i-1}, d_{n, i}\right)\right\}, i \leq 0$, do not depend on $T_{n, 0}$ and are i.i.d., with the same distribution as $\left(t_{1}, d_{1}\right)$.

Let $b_{0}=\mathbb{E}\left|T_{0}\right|$. For $\varepsilon \in(0,1)$, consider the following events:

$$
H_{n}=\left\{n-T_{n, 0} \leq b_{0}(1+\varepsilon)\right\} \cap \bigcap_{i \leq 0}\left\{T_{n, i}-T_{n, i-1} \leq b(1+\varepsilon), d_{n, i} \geq a(1-\varepsilon)\right\} .
$$

These events form a stationary ergodic sequence and, by the strong law of large numbers, have a positive probability for any $\varepsilon>0$. Furthermore, one can see that, for $n^{\prime}<n$, if the events $H_{n^{\prime}}$ and $F_{n^{\prime}}^{(1)}$ occur, with $T_{n, j}=n^{\prime}$ for some $j \leq 0$, then, for event $H_{n}$ to occur, it is sufficient for (34) to hold only for $j$ between $i$ and 0 . Namely,

$$
H_{n^{\prime}} \cap F_{n^{\prime}}^{(1)} \cap\left\{T_{n, j}=n^{\prime}\right\} \cap H_{n}=H_{n^{\prime}} \cap F_{n^{\prime}}^{(1)} \cap\left\{T_{n, j}=n^{\prime}\right\} \cap E_{n^{\prime}, n},
$$

where the event $E_{n^{\prime}, n}$ belongs to the $\sigma$-algebra $\sigma_{n^{\prime}, n}$ and does not depend on $j$. Let $\hat{A}_{n}=$ $H_{n} \cap F_{n}^{(1)}$. Taking the union in all $j$ in (35), we obtain condition (10) with $\hat{A}_{n}$ in place of $A_{n}$. Condition (9), again with $\hat{A}_{n}$ in place of $A_{n}$, may be verified similarly.

Let the constants $c_{-j}, j \geq 0$, be defined as

$$
c_{-j}=0 \quad \text { for } 0 \leq j \leq b_{0}(1+\varepsilon)+b(1+\varepsilon)
$$

and, for $r \geq 1$,

$$
c_{-j}=r a(1-\varepsilon) \quad \text { for }(1+\varepsilon)\left(b_{0}+r b\right)<j \leq(1+\varepsilon)\left(b_{0}+(r+1) b\right) .
$$

Introduce now a second 'future' event

$$
F_{n}^{(2)}=\left\{l_{n+1,0} \geq \sup _{j>0}\left(l_{n+1,-j}+c_{n,-j}\right)\right\} \cap\left\{\text { for all } i>1, l_{n+i, v_{n+i}} \geq \sup _{j>i}\left(l_{n+1,-j}+c_{n,-j}\right)\right\} .
$$

Clearly, for each $n$, the events $H_{n}, F_{n}^{(1)}$, and $F_{n}^{(2)}$ are mutually independent. Furthermore, the events $F_{n}^{(2)}$ form a stationary ergodic sequence and, by (33), have a strictly positive probability. The meaning of the event $F_{n}^{(2)}$ is: all locations for 'new' particles (with numbers greater than $n$ ) generated by 'old' particles (with numbers less than $n$ ) are relatively small; thus, given the simultaneous occurrence of the three events $H_{n}, F_{n}^{(1)}$, and $F_{n}^{(2)}$, all future links (starting from time $n$ ) are established only between particles numbered $n, n+1, n+2, \ldots$.

Let $F_{n}=F_{n}^{(1)} \cap F_{n}^{(2)}$, and let $A_{n}=H_{n} \cap F_{n}$. We may conclude that the stationary sequence of events $\left\{F_{n}\right\}$ satisfy properties (F1) and (F2). Furthermore, an extra intersection with events $F_{n}^{(2)}$ preserves properties (10) and (9), so the conclusion of Corollary 2 holds.

The conclusions of Theorem 1 thus hold. It is also easy to verify aperiodicity for the times $\tau_{n}$ defined in Theorem 1 , because $\mathbb{P}\left(\tau_{2}=1\right)>0$. Then we may take the random functions $R_{i}$ of Section 2 to be given by $R_{i}\left(X_{n+i}, X_{n}\right)=\left(X_{n,-j}, \ldots, X_{n, 0}\right)$ for any fixed $j$, and conclude that the conditions of Theorem 2 and Corollary 3 are satisfied too. The result now follows from the latter corollary.

\section{Relation to Harris ergodicity}

In this section we revisit the basic concept of Harris ergodicity and show that it may be considered as a particular case of the approach developed in Section 2. 
It is known (see, e.g. [6], [7], and [18]) that a time-homogeneous Markov chain $\left\{X_{n}\right\}$ taking values in a measurable state space $\left(\mathcal{X}, \mathscr{B}_{X}\right)$ may be represented as a stochastic recursion (1) with i.i.d. driving sequence $\left\{\xi_{n}\right\}$ if one assumes $\mathscr{B}_{X}$ to be countably generated. Moreover, without loss of generality, one may assume that the random variables $\xi_{n}$ are real valued and uniformly distributed on the interval $(0,1)$.

Recall the following classical definition.

Definition. A time-homogeneous aperiodic Markov chain $\left\{X_{n}\right\}$ taking values in a general state space $(\mathcal{X}, \mathcal{B} X)$ is Harris ergodic (or Harris positive recurrent) if the following conditions hold:

(i) there exist a set $V \in \mathcal{B}_{X}$, a number $0<p \leq 1$, an integer $m \geq 1$, and a probability measure $\varphi$ on $\left(\mathcal{X}, \mathcal{B}_{\mathcal{X}}\right)$ such that

(i.1) if $\tau \equiv \tau(V):=\min \left\{n \geq 1: X_{n} \in V\right\}$ is the first hitting time of the set $V$ then, for any $x \in \mathcal{X}$,

$$
\mathbb{P}_{x}(\tau<\infty)=1,
$$

(i.2) $\sup _{x \in V} \mathbb{E}_{x} \tau<\infty$,

(ii) for any $x \in V$,

$$
\mathbb{P}_{x}\left(X_{m} \in \cdot\right) \geq p \varphi(\cdot),
$$

where $\mathbb{P}_{x}$ and $\mathbb{E}_{x}$ respectively denote the probability and expectation conditional on $\left\{X_{0}=x\right\}$. Note that, frequently, the set $V$ is called positive recurrent if conditions (i.1)-(i.2) hold.

The following result holds (see, e.g. [22]).

Proposition 3. Assume that the Markov chain $\left\{X_{n}\right\}$ is Harris ergodic. Then there exists a unique stationary (invariant) distribution $\pi$, which is also limiting in the sense of convergence in the total variation norm: for any $X_{0}=x \in \mathcal{X}$,

$$
\sup _{B \in \mathscr{B}_{x}}\left|\mathbb{P}_{x}\left(X_{n} \in B\right)-\pi(B)\right| \rightarrow 0 \text { as } n \rightarrow \infty .
$$

Conversely, if (36) holds for any initial value $X_{0}=x \in \mathcal{X}$ then the Markov chain is Harris ergodic.

The 'coupling-type' interpretation of the dynamics of a Harris ergodic Markov chain was proposed in [2] and [24]; see also [6], [7], and [14]. This may be done as follows: we run a Markov chain until it hits the set $V$ (say at time $n$ ), then we flip a coin (independently of everything else) with probability $p$ of getting a head. If this happens then we say that $n+m$ is the success time when the Markov chain 'forgets its past', i.e. $X_{n+m}$ has distribution $\varphi$ which is independent of what has happened before time $n$ (but may depend on what has happened at times $n+1, \ldots, n+m-1$ ). If, on the contrary, we get a tail (which occurs with probability $1-p$ ), we wait for the first time after time $n+m$ when the Markov chain visits $V$ again and then independently flip another coin. After a geometric number of trials, we come to a success with probability 1. It is well known (see, e.g. [1] or [22]) that the Harris ergodic Markov chain may be made regenerative if $m=1$, and wide-sense regenerative and possessing the one-dependence property if $m \geq 2$ (the definitions are given in Section 2 after Theorem 4). More precisely, let $0=T_{0}<T_{1}<T_{2}<\cdots$ be the times of successes. Then the cycle lengths $T_{i+1}-T_{i}$ are i.i.d. in $i \geq 0$, and the cycles $\left(T_{i+1}-T_{i}, X_{T_{i}}, X_{T_{i}+1}, \ldots, X_{T_{i+1}-1}\right)$ are i.i.d. in $i \geq 1$ if $m=1$, and are one-dependent and identically distributed (for $i \geq 1$ ) if $m \geq 2$. 
The one-dependence follows since if $m \geq 2$ then the set $\{n+1, \ldots, n+m-1\}$ is nonempty, and if, say, $T_{i}=n+m$ for some $i$, then the values $X_{n+1}, \ldots, X_{n+m-1}$ belong to the $i$ th cycle, but they also depend, in general, on the value $X_{n+m}$ that belongs to the $(i+1)$ th cycle.

For self-containedness, we recall in more detail the coupling construction of Athreya and Ney [2], in the particular case $m=1$; see [27, p. 366] for the general case. Let $P(x, B)$ be the transition kernel of the Markov chain. Then, using condition (2) in the above definition of Harris ergodicity, for $x \in V$,

$$
P(x, B)=p \varphi(B)+(1-p) \frac{P(x, B)-p \varphi(B)}{1-p} \equiv p \varphi(B)+(1-p) Q(x, B),
$$

where $Q$ is also a transition probability kernel.

Now we provide the coupling construction. First, let $\left\{\alpha_{n}\right\}$ be an i.i.d. sequence with common distribution $\mathbb{P}\left(\alpha_{n}=1\right)=1-\mathbb{P}\left(\alpha_{n}=0\right)=p$. Second, let $\left\{\zeta_{n}\right\}$ be another i.i.d. sequence (having, say, a distribution which is uniform on $(0,1)$ ) that does not depend on $\left\{\alpha_{n}\right\}$. Furthermore, let $g_{1}: \mathcal{X} \times(0,1) \rightarrow \mathcal{X}$ and $g_{2}: V \times(0,1) \rightarrow \mathcal{X}$ be two measurable functions such that $g_{1}\left(x, \zeta_{n}\right)$ has distribution $P(x, \cdot)$ and $g_{2}\left(x, \zeta_{n}\right)$ has distribution $Q(x, \cdot)$. Finally, let $\left\{\psi_{n}\right\}$ be a third independent i.i.d. sequence with distribution $\varphi$.

Then the dynamics of $X_{n}$ are defined as follows:

- if $X_{n} \in V$ and $\alpha_{n+1}=1$, then $X_{n+1}=\psi_{n+1}$;

- if $X_{n} \in V$ and $\alpha_{n+1}=0$, then $X_{n+1}=g_{2}\left(x, \zeta_{n+1}\right)$;

- if $X_{n} \in \bar{V}$ then $X_{n+1}=g_{1}\left(x, \zeta_{n+1}\right)$.

Clearly, $X_{n}$ may be represented as a stochastic recursion with an i.i.d. driving sequence $\xi_{n}=$ $\left(\zeta_{n}, \alpha_{n}, \psi_{n}\right)$. Therefore, for $m=1$, Proposition 3 may be viewed as a particular case of Corollary 3, with $H_{n}=\left\{X_{n} \in V\right\}, F_{n}=\left\{\alpha_{n+1}=1\right\}, A_{n}=H_{n} \cap F_{n}, \tau_{n}=T_{n}$, and $R_{i}\left(X_{\tau_{n}+i}, X_{\tau_{n}}\right)=X_{\tau_{n}+i}$. This follows since condition (7) and then conditions (9) and (10) are immediately verified.

In the case $m>1$, we need a slightly more elaborated coupling construction to conclude that again Proposition 3 may be viewed as a particular case of Corollary 3.

\section{Comments}

There is an extensive list of other examples, and we mention here a few only.

First, there are directions where the methodology may be applied directly: Markov chains with long memory (see, e.g. [9], [11], and [16]); excited random walks (see, e.g. [4], [5], and [21]); modified random walks (see, e.g. [25]).

Second, there are models which involve conditioning on the infinite future which-in contrast with examples considered in this paper-has probability 0 in the original probability space; see, e.g. [3].

A further interesting example of an embedded regenerative structure is of shifts of Brownian motions; see [20].

In the case where the future event $F_{n}$ admits a representation

$$
F_{n}=\bigcap_{k \geq n} F_{n, k}
$$

where the $F_{n, k} \in \sigma_{n, k}$ satisfy the monotonicity property (7), one can introduce a general scheme for 'perfect simulation' of the process along the lines of, say, [13]. 
We also comment that our results may be directly extended onto more general models where either the elements of the driving sequence $\left\{\xi_{n}\right\}$ remain independent but are only 'asymptotically identically distributed', or where this sequence is regenerative or, more generally, converges (in an appropriate manner) to a regenerative sequence. Here the renovation method (see, e.g. [6]), or the method of renovating events, may be of use.

\section{Acknowledgements}

The authors are grateful to the anonymous referees for their insightful and helpful comments.

\section{References}

[1] Asmussen, S. (2003). Applied Probability and Queues. 2nd edn. Springer, New York.

[2] Athreya, K. B. AND Ney, P. A. (1978). A new approach to the limit theory of recurrent Markov chains. Trans. Amer. Math. Soc. 245, 493-501.

[3] Benjamini, I. And Berestycki, N. (2010). Random paths with bounded local time. J. Europ. Math. Soc. 12, 819-854.

[4] Benjamini, I. And Wilson, D. B. (2003). Excited random walk. Electron. Commun. Probab. 8, 86-92.

[5] Bérard, J. AND Ramírez, A. (2007). Central limit theorem for the excited random walk in dimension $D \geq 2$. Electron Commun. Prob. 12, 303-314.

[6] Borovkov, A. A. (1998). Ergodicity and Stability of Stochastic Processes. John Wiley, Chichester.

[7] Borovkov, A. A. AND Foss, S. G. (1992). Stochastically recursive sequences and their generalizations. Siberian Adv. Math. 2, 16-81.

[8] Chernysh, K and Ramasmami, S. (2013). Optimal deterministic algorithms and Markov evolution for the infinite bin model. Working paper.

[9] Comets, F., Fernández, R. and Ferrari, P. A. (2002). Processes with long memory: regenerative construction and perfect simulation. Ann. Appl. Prob. 12, 921-943.

[10] Denisov, D., Foss, S. and Konstantopoulos, T. (2012). Limit theorems for a random directed slab graph. Ann. Appl. Prob. 22, 702-733.

[11] De Santis, E. And Piccioni, M. (2012). Backward coalescence times for perfect simulation of chains with infinite memory. J. Appl. Prob. 49, 319-337.

[12] Durrett, R. and Schinazi, R. B. (2000). Boundary modified contact processes. J. Theoret. Prob. 13, $575-594$.

[13] Foss, S. and Konstantopoulos, T. (2003). Extended renovation theory and limit theorems for stochastic ordered graphs. Markov Process. Relat. Fields 9, 413-468.

[14] Foss, S. And Konstantopoulos, T. (2004). An overview of some stochastic stability methods. J. Operat. Res. Soc. Japan 47, 275-303.

[15] Foss, S., Martin, J. And Schmidt, P. (2013). Long-range last-passage percolation on the line. To appear in Ann. Appl. Prob.

[16] Gallo, S. (2011). Chains with unbounded variable length memory: perfect simulation and a visible regeneration scheme. Adv. Appl. Prob. 43, 735-759.

[17] Galves, A. and Presutti, E. (1987). Edge fluctuations for the one-dimensional supercritical contact process. Ann. Prob. 15, 1131-1145.

[18] KIFER, Y. (1986). Ergodic Theory of Random Transformations. Birkhäuser, Boston, MA.

[19] Kuczek, T. (1989). The central limit theorem for the right edge of supercritical oriented percolation. Ann. Prob. 17, 1322-1332.

[20] Last, G., Möerters, P. and Thorisson, H. (2013). Unbiased shifts of Brownian motion. Submitted. Available at http://arxiv.org/abs/1112.5373v1.

[21] Menshikov, M., Popov, S., Ramirez, A. F. and Vachkovskaia, M. (2012). On a general many-dimensional excited random walk. Ann. Appl. Prob. 40, 2106-2130.

[22] Meyn, S. P. ANd Tweedie, R. L. (1993). Markov Chains and Stochastic Stability. Springer, London.

[23] Mountford, T. S. And SweEt, T. D. (2000). An extension of Kuczek's argument to nonnearest neighbor contact processes. J. Theoret. Prob. 13, 1061-1081.

[24] Nummelin, E. (1978). A splitting technique for Harris recurrent Markov chains. Z. Wahrscheinlichkeitsth. 43, 309-318.

[25] Raimond, O. AND Schapira, B. (2012). Random walks with occasionally modified transition probabilities. Submitted. Available at http://arxiv.org/abs/0911.3886v2.

[26] Stacey, A. M. (2003). Partial immunization processes. Ann. Appl. Prob. 13, 669-690.

[27] Thorisson, H. (2000). Coupling, Stationarity, and Regeneration. Springer, New York.

[28] Tzioufas, A. (2011). On the growth of the one-dimensional reverse immunization contact processes. J. Appl. Prob. 48, 611-623. 